\title{
Some criteria of boundedness of $L$-index in a direction for slice holomorphic functions of several complex variables
}

\author{
Andriy Bandura, Oleh SKaskiv \\ (Presented by I. I. Skrypnik) \\ The paper is devoted to the 100th anniversary \\ of Georgii Dmitrievich Suvorov
}

\begin{abstract}
In this paper, we investigate slice holomorphic functions of several complex variables having bounded $L$-index in a direction, i.e. we consider functions which are entire on every slice $\left\{z^{0}+t \mathbf{b}: t \in\right.$ $\mathbb{C}\}$ for every $z^{0} \in \mathbb{C}^{n}$ and for a given direction $\mathbf{b} \in \mathbb{C}^{n} \backslash\{\mathbf{0}\}$. For this function class we prove some criteria of boundedness of $L$-index in the direction describing local behavior of maximum modulus, minimum modulus of the slice holomorphic function and providing estimates of logarithmic derivative and distribution of zeros. Moreover, we obtain analogs of known Hayman's theorem and logarithmic criteria. They are applicable in analytic theory of differential equations. We also investigate value distribution of these functions and present existence theorem for this function class. It indicates that bounded multiplicity of zeros for slice holomorphic function $F: \mathbb{C}^{n} \rightarrow \mathbb{C}$ is the necessary and sufficient condition for existence of a positive continuous function $L: \mathbb{C}^{n} \rightarrow \mathbb{R}_{+}$ such that $F$ has bounded $L$-index in the direction.
\end{abstract}

2010 MSC. 32A10, 32A17, 32A37, 30H99, 30A05.

Key words and phrases. Bounded index, bounded $L$-index in direction, slice function, holomorphic function, maximum modulus, minimum modulus, bounded $l$-index, existence theorem, distribution of zeros, logarithmic derivative, directional derivative.

\section{Introduction}

The paper is addendum to [1]. There was introduced a concept of $L$ index boundedness in direction for slice entire functions of several complex

Received 09.05.2019

The authors are thankful to Professor S. Yu. Favorov (Kharkiv) for the formulation of interesting problem. 
variables. Also there was deduced some criteria of $L$-index boundedness in direction. Here we present some applications of these criteria to obtain more useful criteria of $L$ - index boundedness in direction. Among them are logarithmic criterion, analog of Hayman's theorem and estimate of minimum modulus. It should be note that the fist two criteria have applications in analytic theory of differential equations. Also we consider value distribution of function belonging to this function class and prove existence theorem. It demonstrates extent of the class, i.e. slice holomorphic functions of bounded $L$-index in direction.

The paper is devoted to the following problem.

Problem 1.1. Is it possible to deduce main facts of theory of entire functions having bounded L-index in the direction $\mathbf{b} \in \mathbb{C}^{n} \backslash\{0\}$ for functions which are holomorphic on the slices $\left\{z^{0}+t \mathbf{b}: t \in \mathbb{C}\right\}$ and are joint continuous?

Let us introduce some notations from [1]. Let $\mathbb{R}_{+}=(0,+\infty), \mathbb{R}_{+}^{*}=$ $[0,+\infty), \mathbf{0}=(0, \ldots, 0), \mathbf{b}=\left(b_{1}, \ldots, b_{n}\right) \in \mathbb{C}^{n} \backslash\{\mathbf{0}\}$ be a given direciton, $L: \mathbb{C}^{n} \rightarrow \mathbb{R}_{+}$be a continuous function, $F: \mathbb{C}^{n} \rightarrow \mathbb{C}$ an entire function. The slice functions on a line $\left\{z^{0}+t \mathbf{b}: t \in \mathbb{C}\right\}$ for fixed $z^{0} \in \mathbb{C}$ we will denote as $g_{z^{0}}(t)=F\left(z^{0}+t \mathbf{b}\right)$ and $l_{z^{0}}(t)=L\left(z^{0}+t \mathbf{b}\right)$.

Let $\widetilde{\mathcal{H}}_{\mathrm{b}}^{n}$ be a class of functions which are holomorphic on every slices $\left\{z^{0}+t \mathbf{b}: t \in \mathbb{C}\right\}$ for each $z^{0} \in \mathbb{C}^{n}$ and let $\mathcal{H}_{\mathbf{b}}^{n}$ be a class of functions from $\widetilde{\mathcal{H}}_{\mathrm{b}}^{n}$ which are joint continuous.

The notation $\partial_{\mathbf{b}} F(z)$ stands for the derivative of the function $g_{z}(t)$ at the point 0 , i.e. for every $p \in \mathbb{N} \quad \partial_{\mathbf{b}}^{p} F(z)=g_{z}^{(p)}(0)$, where $g_{z}(t)=$ $F(z+t \mathbf{b})$ is entire function of complex variable $t \in \mathbb{C}$ for given $z \in \mathbb{C}^{n}$. In this research, we will often call this derivative as directional derivative because if $F$ is entire function in $\mathbb{C}^{n}$ then the derivatives of the function $g_{z}(t)$ matches with directional derivatives of the function $F$. Together the hypothesis on joint continuity and the hypothesis on holomorphy in one direction do not imply holomorphy in whole $n$-dimensional complex space. We give some examples to demonstrate it. For $n=2$ let $f$ : $\mathbb{C} \rightarrow \mathbb{C}$ be an entire function, $g: \mathbb{C} \rightarrow \mathbb{C}$ be a continuous function. Then $f\left(z_{1}\right) g\left(z_{2}\right), f\left(z_{1}\right) \pm g\left(z_{2}\right), f\left(z_{1} \cdot g\left(z_{2}\right)\right)$ are functions which are holomorphic in the direction $(1,0)$ and are joint continuous in $\mathbb{C}^{2}$. Moreover, if we have performed an affine transformation

$$
\left\{\begin{array}{l}
z_{1}=b_{2} z_{1}^{\prime}+b_{1} z_{2}^{\prime}, \\
z_{2}=b_{2} z_{1}^{\prime}-b_{1} z_{2}^{\prime}
\end{array}\right.
$$

then the appropriate new functions are also holomorphic in the direction $\left(b_{1}, b_{2}\right)$ and are joint continuous in $\mathbb{C}^{2}$, where $b_{1} \neq 0, b_{2} \neq 0$. 
A function $F \in \mathcal{H}_{\mathrm{b}}^{n}$ is said [1] to be of bounded $L$-index in the direction $\mathbf{b}$, if there exists $m_{0} \in \mathbb{Z}_{+}$such that for all $m \in \mathbb{Z}_{+}$and each $z \in \mathbb{C}^{n}$ inequality

$$
\frac{\left|\partial_{\mathbf{b}}^{m} F(z)\right|}{m ! L^{m}(z)} \leq \max _{0 \leq k \leq m_{0}} \frac{\left|\partial_{\mathbf{b}}^{k} F(z)\right|}{k ! L^{k}(z)},
$$

is true. The least such integer number $m_{0}$, obeying (1.1), is called the $L$-index in the direction $\mathbf{b}$ of the function $F$ and is denoted by $N_{\mathbf{b}}(F, L)$. If such $m_{0}$ does not exist, then we put $N_{\mathbf{b}}(F, L)=\infty$, and the function $F$ is called of unbounded $L$-index in the direction $\mathbf{b}$ in this case. If $L(z) \equiv 1$, then the function $F$ is said to be of bounded index in the direction $\mathbf{b}$ and $N_{\mathbf{b}}(F)=N_{\mathbf{b}}(F, 1)$ is called the index in the direction $\mathbf{b}$. For $n=1, \mathbf{b}=1, L(z)=l(z), z \in \mathbb{C}$ inequality (1.1) defines a function of bounded $l$-index with the $l$-index $N(F, l) \equiv N_{1}(F, l)[19,20]$, and if in addition $l(z) \equiv 1$, then we obtain a definition of index boundedness with index $N(F) \equiv N_{1}(F, 1)[21,22]$. It is also worth to mention paper [31], which introduces the concept of generalized index. It is quite close to the bounded $l$-index. Let $N_{\mathbf{b}}\left(F, L, z^{0}\right)$ stands for the $L$-index in the direction $\mathbf{b}$ of the function $F$ at the point $z^{0}$, i.e., it is the least integer $m_{0}$, for which inequality (1.1) is satisfied at this point $z=z^{0}$. By analogy, the notation $N\left(f, l, z^{0}\right)$ is defined if $n=1$, i.e. in the case of functions of one variable.

Note that the positivity and continuity of the function $L$ are weak restrictions to deduce constructive results. Thus, we assume additional restrictions by the function $L$.

Let us denote

$$
\begin{aligned}
& \lambda_{\mathbf{b}}(\eta) \\
= & \sup _{z \in \mathbb{C}^{n}} \sup _{t_{1}, t_{2} \in \mathbb{C}}\left\{\frac{L\left(z+t_{1} \mathbf{b}\right)}{L\left(z+t_{2} \mathbf{b}\right)}:\left|t_{1}-t_{2}\right| \leq \frac{\eta}{\min \left\{L\left(z+t_{1} \mathbf{b}\right), L\left(z+t_{2} \mathbf{b}\right)\right\}}\right\} .
\end{aligned}
$$

By $Q_{\mathrm{b}}^{n}$ we denote a class of positive continuous function $L: \mathbb{C}^{n} \rightarrow \mathbb{R}_{+}$, satisfying the condition

$$
(\forall \eta \geq 0): \lambda_{\mathbf{b}}(\eta)<+\infty
$$

Moreover, it is sufficient to require validity of (1.2) for one value $\eta>0$.

For a positive continuous function $l(t), t \in \mathbb{C}$, and $\eta>0$ we define $\lambda(\eta) \equiv \lambda_{1}^{\mathbf{b}}(\eta)$ in the cases when $\mathbf{b}=1, n=1, L \equiv l$. As in [26], let $Q \equiv Q_{1}^{1}$ be a class of positive continuous functions $l(t), t \in \mathbb{C}$, obeying the condition $0<\lambda(\eta)<+\infty$ for all $\eta>0$.

Besides, we denote by $\langle a, c\rangle=\sum_{j=1}^{n} a_{j} \overline{c_{j}}$ the Hermitian scalar product in $\mathbb{C}^{n}$, where $a, c \in \mathbb{C}^{n}$. 


\section{Auxiliary propositions}

Let $L^{*}(z)$ be a positive continuous function in $\mathbb{C}^{n}$. We denote $L \asymp L^{*}$, if for some $\theta_{1}, \theta_{2}, 0<\theta_{1} \leq \theta_{2}<+\infty$, and for all $z \in \mathbb{C}^{n}$ the following inequalities hold $\theta_{1} L(z) \leq L^{*}(z) \leq \theta_{2} L(z)$.

Proposition 2.1. ([1]) Let $L \in Q_{\mathrm{b}}^{n}, L \asymp L^{*}$. A function $F \in \widetilde{\mathcal{H}}_{\mathbf{b}}^{n}$ has bounded $L^{*}$-index in the direction $\mathbf{b}$ if and only if $F$ is of bounded $L$-index in the direction $\mathbf{b}$.

Theorem 2.1. ([1]) Let $L \in Q_{\mathbf{b}}^{n}$. A function $F \in \widetilde{\mathcal{H}}_{\mathbf{b}}^{n}$ is of bounded $L$-index in the direction $\mathbf{b}$ if and only if for each $\eta>0$ there exist $n_{0}=$ $n_{0}(\eta) \in \mathbb{Z}_{+}$and $P_{1}=P_{1}(\eta) \geq 1$ such that for every $z \in \mathbb{C}^{n}$ there exists $k_{0}=k_{0}(z) \in \mathbb{Z}_{+}, 0 \leq k_{0} \leq n_{0}$, and

$$
\max \left\{\left|\partial_{\mathbf{b}}^{k_{0}} F(z+t \mathbf{b})\right|:|t| \leq \frac{\eta}{L(z)}\right\} \leq P_{1}\left|\partial_{\mathbf{b}}^{k_{0}} F(z)\right| .
$$

Theorem 2.2 ([1]). Let $D$ be an arbitrary bounded domain in $\mathbb{C}^{n}, \mathbf{b} \in$ $\mathbb{C}^{n} \backslash\{\mathbf{0}\}$ be arbitrary direction. If $L: \mathbb{C}^{n} \rightarrow \mathbb{R}_{+}$is continuous function, $F \in \mathcal{H}_{\mathbf{b}}^{n}$ and $(\forall p \in \mathbb{N}) \partial_{\mathbf{b}}^{p} F \in \mathcal{H}_{\mathbf{b}}^{n}$ and $\left(\forall z^{0} \in \bar{D}\right): \quad F\left(z^{0}+t \mathbf{b}\right) \not \equiv 0$, then $N_{\mathbf{b}}(F, L, D)<\infty$.

\section{Estimate of maximum modulus by minimum modulus}

Using Theorem 2.1, we will prove the next criterion of $L$-index boundedness in direction. Similar results was firstly deduced by G. H. Fricke [15] for entire functions of bounded index. Further it was generalized for various classes of holomorphic functions $[4,25,26]$.

Theorem 3.1. Let $L \in Q_{\mathrm{b}}^{n}$. A function $F \in \widetilde{\mathcal{H}}_{\mathbf{b}}^{n}$ has bounded L-index in the direction $\mathbf{b}$ if and only if for any $r_{1}>0, r_{2}>0\left(r_{1}<r_{2}\right)$, there exists $P_{1}=P_{1}\left(r_{1}, r_{2}\right) \geq 1$ such that for every $z^{0} \in \mathbb{C}^{n}$

$$
\max \left\{\left|F\left(z^{0}+t \mathbf{b}\right)\right|:|t|=\frac{r_{2}}{L\left(z^{0}\right)}\right\} \leq P_{1} \max \left\{\left|F\left(z^{0}+t \mathbf{b}\right)\right|:|t|=\frac{r_{1}}{L\left(z_{0}\right)}\right\} .
$$

Proof. Our proof is based on the proof of appropriate theorem for entire functions of bounded $L$-index in direction $[6,8]$.

Necessity. Let $N_{\mathbf{b}}(F, L)<+\infty$. On the contrary, suppose that there exist number $r_{1}$ and $r_{2}, 0<r_{1}<r_{2}$, such that for each $P_{*} \geq 1$ there exists $z^{*}=z^{*}\left(P_{*}\right) \in \mathbb{C}^{n}$ satisfying inequality

$$
\max \left\{\left|F\left(z^{*}+t \mathbf{b}\right)\right|:|t|=\frac{r_{2}}{L\left(z^{*}\right)}\right\}>P_{*} \max \left\{\left|F\left(z^{*}+t \mathbf{b}\right)\right|:|t|=\frac{r_{1}}{L\left(z^{*}\right)}\right\} .
$$


By Theorem 2.1 there exist $n_{0}=n_{0}\left(r_{2}\right) \in \mathbb{Z}_{+}$and $P_{0}=P_{0}\left(r_{2}\right) \geq 1$ such that for all $z^{*} \in \mathbb{C}^{n}$ and some $k_{0}=k_{0}\left(t^{*}, z^{*}\right) \in \mathbb{Z}_{+}, 0 \leq k_{0} \leq n_{0}$, the following inequality holds

$$
\max \left\{\left|\partial_{\mathbf{b}}^{k_{0}} F\left(z^{*}+t \mathbf{b}\right)\right|:|t|=r_{2} / L\left(z^{*}\right)\right\} \leq P_{0}\left|\partial_{\mathbf{b}}^{k_{0}} F\left(z^{*}\right)\right| .
$$

One should observe that for $k_{0}=0$ the proof of necessity is obvious, because (3.2) implies $\max \left\{\left|F\left(z^{*}+t \mathbf{b}\right)\right|:|t|=r_{2} / L\left(z^{*}\right)\right\} \leq P_{0}\left|F\left(z^{*}\right)\right| \leq$ $P_{0} \max \left\{\left|F\left(z^{*}+t \mathbf{b}\right)\right|:|t|=r_{1} / L\left(z^{*}\right)\right\}$.

Suppose that $k_{0}>0$ and

$$
P_{*}=n_{0} !\left(\frac{r_{2}}{r_{1}}\right)^{n_{0}}\left(P_{0}+\frac{r_{1}}{r_{2}-r_{1}}\right)+1
$$

We choose $t_{0} \in \mathbb{C}$ such that $\left|t_{0}\right|=r_{1} / L\left(z^{*}\right)$ and

$$
\left|F\left(z^{*}+t_{0} \mathbf{b}\right)\right|=\max \left\{\left|F\left(z^{*}+t \mathbf{b}\right)\right|:|t|=r_{1} / L\left(z^{*}\right)\right\}>0,
$$

and $t_{0 j} \in \mathbb{C},\left|t_{0 j}\right|=r_{2} / L\left(z^{*}\right)$, be such that

$$
\left|\partial_{\mathbf{b}}^{j} F\left(z^{*}+t_{0 j} \mathbf{b}\right)\right|=\max \left\{\left|\partial_{\mathbf{b}}^{j} F\left(z^{*}+t \mathbf{b}\right)\right|:|t|=r_{2} / L\left(z^{*}\right)\right\},
$$

$j \in \mathbb{Z}_{+}$. In the case $\left|F\left(z^{*}+t_{0} \mathbf{b}\right)\right|=0$ by uniqueness theorem for all $t \in \mathbb{C}$ one has $F\left(z^{*}+t \mathbf{b}\right)=0$. But it contradicts inequality (3). By Cauchy's inequality we deduce

$$
\begin{gathered}
\frac{\left|\partial_{\mathbf{b}}^{j} F\left(z^{*}\right)\right|}{j !} \leq\left(\frac{L\left(z^{*}\right)}{r_{1}}\right)^{j}\left|F\left(z^{*}+t_{0} \mathbf{b}\right)\right|, j \in \mathbb{Z}_{+} \\
\left|\partial_{\mathbf{b}}^{j} F\left(z^{*}+t_{0 j} \mathbf{b}\right)-\partial_{\mathbf{b}}^{j} F\left(z^{*}\right)\right|=\left|\int_{0}^{t_{0 j}} \partial_{\mathbf{b}}^{j+1} F\left(z^{*}+t \mathbf{b}\right) d t\right| \\
\leq\left|\partial_{\mathbf{b}}^{j+1} F\left(z^{*}+t_{0(j+1)} \mathbf{b}\right)\right| \frac{r_{2}}{L\left(z^{*}\right)} .
\end{gathered}
$$

From inequalities (3.4) and (3.5) it follows that

$$
\begin{gathered}
\left|\partial_{\mathbf{b}}^{j+1} F\left(z^{*}+t_{0(j+1)} \mathbf{b}\right)\right| \geq \frac{L\left(z^{*}\right)}{r_{2}}\left\{\left|\partial_{\mathbf{b}}^{j} F\left(z^{*}+t_{0 j} \mathbf{b}\right)\right|-\left|\partial_{\mathbf{b}}^{j} F\left(z^{*}\right)\right|\right\} \\
\geq \frac{L\left(z^{*}+t^{*} \mathbf{b}\right)}{r_{2}}\left|\partial_{\mathbf{b}}^{j} F\left(z^{*}+t_{0 j} \mathbf{b}\right)\right|-\frac{j ! L^{j+1}\left(z^{*}\right)}{r_{2}\left(r_{1}\right)^{j}}\left|F\left(z^{*}+t_{0} \mathbf{b}\right)\right|,
\end{gathered}
$$


$j \in \mathbb{Z}_{+}$. Hence, for $k_{0} \geq 1$ we obtain

$$
\begin{gathered}
\left|\partial_{\mathbf{b}}^{k_{0}} F\left(z^{*}+t_{0 k_{0}} \mathbf{b}\right)\right| \geq \frac{L\left(z^{*}\right)}{r_{2}}\left|\partial_{\mathbf{b}}^{k_{0}-1} F\left(z^{*}+t_{0\left(k_{0}-1\right)} \mathbf{b}\right)\right| \\
-\frac{\left(k_{0}-1\right) ! L^{k_{0}}\left(z^{*}\right)}{r_{2}\left(r_{1}\right)^{k_{0}-1}}\left|F\left(z^{*}+t_{0} \mathbf{b}\right)\right| \geq \ldots \geq \frac{L^{k_{0}}\left(z^{*}\right)}{\left(r_{2}\right)^{k_{0}}}\left|F\left(z^{*}+t_{00} \mathbf{b}\right)\right| \\
-\left(\frac{0 !}{\left(r_{2}\right)^{k_{0}}}+\frac{1 !}{\left(r_{2}\right)^{k_{0}-1} r_{1}}+\ldots+\frac{\left(k_{0}-1\right) !}{r_{2}\left(r_{1}\right)^{k_{0}-1}}\right) L^{k_{0}}\left(z^{*}\right)\left|F\left(z^{*}+t_{0} \mathbf{b}\right)\right| \\
=\frac{L^{k_{0}}\left(z^{*}\right)}{\left(r_{2}\right)^{k_{0}}}\left|F\left(z^{*}+t_{0} \mathbf{b}\right)\right|\left(\frac{\left|F\left(z^{*}+t_{00} \mathbf{b}\right)\right|}{\left|F\left(z^{*}+t_{0} \mathbf{b}\right)\right|}-\sum_{j=0}^{k_{0}-1} j !\left(\frac{r_{2}}{r_{1}}\right)^{j}\right) .
\end{gathered}
$$

From (3) one has $\left|F\left(z^{*}+t_{00} \mathbf{b}\right)\right| /\left|F\left(z^{*}+t_{0} \mathbf{b}\right)\right|>P_{*}$. Besides, the following inequality is true

$$
\sum_{j=0}^{k_{0}-1} j !\left(\frac{r_{2}}{r_{1}}\right)^{j} \leq k_{0} !\left(\frac{\left(r_{2} / r_{1}\right)^{k_{0}}-1}{r_{2} / r_{1}-1}\right) \leq n_{0} ! \frac{r_{1}}{r_{2}-r_{1}}\left(\frac{r_{2}}{r_{1}}\right)^{n_{0}} .
$$

Applying (3.3), we get

$$
\frac{\left|F\left(z^{*}+t_{00} \mathbf{b}\right)\right|}{\left|F\left(z^{*}+t_{0} \mathbf{b}\right)\right|}-\sum_{j=0}^{k_{0}-1} j ! \frac{r_{2}^{j}}{r_{1}^{j}}>P_{*}-\frac{n_{0} ! r_{1}}{r_{2}-r_{1}}\left(\frac{r_{2}}{r_{1}}\right)^{n_{0}}=n_{0} !\left(\frac{r_{2}}{r_{1}}\right)^{n_{0}} P_{0}+1 .
$$

In view of (3.2) and (3.4), from (3.6) it follows that

$$
\begin{gathered}
\left|\partial_{\mathbf{b}}^{k_{0}} F\left(z^{*}+t_{0 k_{0}} \mathbf{b}\right)\right|>\frac{L^{k_{0}}\left(z^{*}\right)}{\left(r_{2}\right)^{k_{0}}}\left(P_{*}-n_{0} ! \frac{r_{1}}{r_{2}-r_{1}}\left(\frac{r_{2}}{r_{1}}\right)^{n_{0}}\right)\left(\frac{r_{1}}{L\left(z^{*}\right)}\right)^{k_{0}} \\
\quad \times \frac{\left|\partial_{\mathbf{b}}^{k_{0}} F\left(z^{*}\right)\right|}{k_{0} !} \geq\left(\frac{r_{1}}{r_{2}}\right)^{n_{0}}\left(P_{*}-n_{0} ! \frac{r_{1}}{r_{2}-r_{1}}\left(\frac{r_{2}}{r_{1}}\right)^{n_{0}}\right) \frac{\left|\partial_{\mathbf{b}}^{k_{0}} F\left(z^{*}+t_{0 k_{0}} \mathbf{b}\right)\right|}{n_{0} ! P_{0}} .
\end{gathered}
$$

Hence, $P_{*}<n_{0} !\left(\frac{r_{2}}{r_{1}}\right)^{n_{0}}\left(P_{0}+\frac{r_{1}}{r_{2}-r_{1}}\right)$, and it contradicts (3.3).

Sufficiency. Choose any $r_{1} \in(0,1)$ and $r_{2} \in(1,+\infty)$. For given $z^{0} \in \mathbb{C}^{n}$ we develop the function $F\left(z^{0}+t \mathbf{b}\right)$ in power series by powers $t$

$$
F\left(z^{0}+t \mathbf{b}\right)=\sum_{m=0}^{\infty} b_{m}\left(z^{0}\right) t^{m}, b_{m}\left(z^{0}\right)=\frac{\partial_{\mathbf{b}}^{m} F\left(z^{0}\right)}{m !}
$$

in the disc $\left\{t:|t| \leq \beta / L\left(z^{0}\right)\right\}$. For $r>0$ we define $M_{\mathbf{b}}\left(r, z^{0}, F\right)=$ $\max \left\{\left|F\left(z^{0}+t \mathbf{b}\right)\right|:|t|=r\right\}, \mu_{\mathbf{b}}\left(r, z^{0}, F\right)=\max \left\{\left|b_{m}\left(z^{0}\right)\right| r^{m}: m \geq 0\right\}$, $\nu_{\mathbf{b}}\left(r, z^{0}, F\right)=\max \left\{\left|b_{m}\left(z^{0}\right)\right| r^{m}:\left|b_{m}\left(z^{0}\right)\right| r^{m}=\mu_{\mathbf{b}}\left(r, z^{0}, F\right)\right\}$. 
By Cauchy's inequality $\mu_{\mathbf{b}}\left(r, z^{0}, F\right) \leq M_{\mathbf{b}}\left(r, z^{0}, F\right)$. But for $r=\frac{1}{L\left(z^{0}\right)}$ one has

$$
M_{\mathbf{b}}\left(r_{1} r, z^{0}, F\right) \leq \sum_{m=0}^{\infty}\left|b_{m}\left(z^{0}\right)\right| r^{m} r_{1}^{m} \leq \mu_{\mathbf{b}}\left(r, z^{0}, F\right) \sum_{m=0}^{\infty} r_{1}^{m}=\frac{\mu_{\mathbf{b}}\left(r, z^{0}, F\right)}{1-r_{1}}
$$

and, applying monotonicity of $\nu_{\mathbf{b}}\left(r, z^{0}, F\right)$ in $r$, we get

$$
\ln \mu_{\mathbf{b}}\left(r_{2} r, z^{0}, F\right)-\ln \mu_{\mathbf{b}}\left(r, z^{0}, F\right)=\int_{r}^{r_{2} r} \frac{\nu_{\mathbf{b}}\left(t, z^{0}, F\right)}{t} d t \geq \nu_{\mathbf{b}}\left(r, z^{0}, F\right) \ln r_{2} .
$$

Hence, it follows that

$$
\begin{array}{r}
\nu_{\mathbf{b}}\left(r, z^{0}, F\right) \leq \frac{1}{\ln r_{2}}\left(\ln \mu_{\mathbf{b}}\left(r_{2} r, z^{0}, F\right)-\ln \mu_{\mathbf{b}}\left(r, z^{0}, F\right)\right) \\
\leq \frac{1}{\ln r_{2}}\left\{\ln M_{\mathbf{b}}\left(r_{2} r, z^{0}, F\right)-\ln \left(\left(1-r_{1}\right) M_{\mathbf{b}}\left(r_{1} r, z^{0}, F\right)\right)\right\} \\
\left.=-\frac{\ln \left(1-r_{1}\right)}{\ln r_{2}}+\frac{1}{\ln r_{2}}\left\{\ln M_{\mathbf{b}}\left(r_{2} r, z^{0}, F\right)-\ln M_{\mathbf{b}}\left(r_{1} r, z^{0}, F\right)\right)\right\}
\end{array}
$$

Let $N_{\mathbf{b}}\left(F, L, z^{0}\right)$ be the $L$-index in direction of the function $F$ at the point $z^{0}$, i.e, $N_{\mathbf{b}}\left(F, L, z^{0}\right)$ is the least number $m_{0}$, for which inequality (1.1) holds at the point $z=z^{0}$. Obviously that $N_{\mathbf{b}}\left(F, L, z^{0}\right) \leq$ $\nu_{\mathbf{b}}\left(1 / L\left(z^{0}\right), z^{0}, F\right)=\nu_{\mathbf{b}}\left(r, z^{0}, F\right)$. But inequality (3.1) can be rewritten in the following form $M_{\mathbf{b}}\left(\frac{r_{2}}{L\left(z^{0}\right)}, z^{0}, F\right) \leq P_{1}\left(r_{1}, r_{2}\right) M_{\mathbf{b}}\left(\frac{r_{1}}{L\left(z^{0}\right)}, z^{0}, F\right)$. Thus, from (3.7) one has $N_{\mathbf{b}}\left(F, L, z^{0}\right) \leq-\frac{\ln \left(1-r_{1}\right)}{\ln r_{2}}+\frac{\ln P_{1}\left(r_{1}, r_{2}\right)}{\ln r_{2}}$ for every $z^{0} \in \mathbb{C}^{n}$, that is $N_{\mathbf{b}}(F, L) \leq-\frac{\ln \left(1-r_{1}\right)}{\ln r_{2}}+\frac{\ln P_{1}\left(r_{1}, r_{2}\right)}{\ln r_{2}}$. Theorem 3.1 is proved.

In view of proof of sufficiency in Theorem 3.1 the following lemma is valid.

Lemma 3.1. Let $L \in Q_{\mathrm{b}}^{n}, F \in \widetilde{\mathcal{H}}_{\mathrm{b}}^{n}$. If there exist numbers $r_{1}$ and $r_{2}$, $0<r_{1}<1<r_{2}$, and $P_{1} \geq 1$ such that for every $z^{0} \in \mathbb{B}^{n}$ inequality (3.1) holds then the function $F$ is of bounded L-index in the direction $\mathbf{b}$.

We can relax sufficient conditions of Lemma 3.1, replacing the condition $0<r_{1}<1<r_{2}<+\infty$ by $0<r_{1}<r_{2}<+\infty$.

Proposition 3.1. Let $L \in Q_{\mathbf{b}}^{n}, F \in \widetilde{\mathcal{H}}_{\mathbf{b}}^{n}$. If there exist $r_{1}$ and $r_{2}, 0<$ $r_{1}<r_{2}<+\infty$, and $P_{1} \geq 1$ such that for all $z^{0} \in \mathbb{C}^{n}$ inequality (3.1) holds, then the function has $F$ bounded $L$-index in the direction $\mathbf{b}$. 
Proof. Our proof uses the idea of A. D. Kuzyk and M. M. Sheremeta [20]. They proposed the method to investigate of $l$-index boundedness of entire solutions of linear differential equations.

Inequality (3.1) for $0<r_{1}<r_{2}<+\infty$ implies

$$
\begin{gathered}
\max \left\{\left|F\left(z^{0}+t \mathbf{b}\right)\right|:|t|=\frac{2 r_{2}}{r_{1}+r_{2}} \frac{r_{1}+r_{2}}{2 L\left(z^{0}\right)}\right\} \\
\leq P_{1} \max \left\{\left|F\left(z^{0}+t \mathbf{b}\right)\right|:|t|=\frac{2 r_{1}}{r_{1}+r_{2}} \frac{r_{1}+r_{2}}{2 L\left(z_{0}\right)}\right\} .
\end{gathered}
$$

Defining $L^{*}(z)=\frac{2 L(z)}{r_{1}+r_{2}}$, we obtain

$$
\begin{gathered}
\max \left\{\left|F\left(z^{0}+t \mathbf{b}\right)\right|:|t|=\frac{2 r_{2}}{\left(r_{1}+r_{2}\right) L^{*}\left(z^{0}\right)}\right\} \\
\leq P_{1} \max \left\{\left|F\left(z^{0}+t \mathbf{b}\right)\right|:|t|=\frac{2 r_{1}}{\left(r_{1}+r_{2}\right) L^{*}\left(z^{0}\right)}\right\},
\end{gathered}
$$

where $0<\frac{2 r_{1}}{r_{1}+r_{2}}<1<\frac{2 r_{2}}{r_{1}+r_{2}}<+\infty$. This means that $F$ has bounded $L^{*}$-index in the direction $\mathbf{b}$. And by Proposition 2.1 the function $F$ has bounded $L$-index in the direction $\mathbf{b}$.

The following theorem gives estimate of maximum modulus by minimum modulus. It was firstly obtained by G. H. Fricke [15] for entire functions of bounded index.

Theorem 3.2. Let $L \in Q_{\mathbf{b}}^{n}$. If the function $F \in \widetilde{\mathcal{H}}_{\mathbf{b}}^{n}$ is of bounded $L$ index in the direction $\mathbf{b}$ then for each $R>0$ there exist $P_{2}(R) \geq 1$ and $\eta(R) \in(0, R)$ such that for every $z^{0} \in \mathbb{C}^{n}$ and some $r=r\left(z^{0}\right) \in[\eta(R), R]$ the inequality holds

$$
\max \left\{\left|F\left(z^{0}+t \mathbf{b}\right)\right|:|t|=r / L\left(z^{0}\right)\right\} \leq P_{2} \min \left\{\left|F\left(z^{0}+t \mathbf{b}\right)\right|:|t|=r / L\left(z^{0}\right)\right\} .
$$

Proof. Our proof is based on the proof of appropriate theorem for entire functions of bounded $L$-index in direction [6].

Let $N_{\mathbf{b}}(F, L)=N<+\infty$ and $R \geq 0$. Put

$$
R_{0}=1, r_{0}=\frac{R}{8(R+1)}, R_{j}=\frac{R_{j-1}}{4 N} r_{j-1}^{N}, r_{j}=\frac{1}{8} R_{j}(j=1,2, \ldots, N) .
$$

Let $z^{0} \in \mathbb{C}^{n}$ and $N_{0}=N_{\mathbf{b}}\left(z^{0}, L, F\right)$ be the $L$-index in the direction $\mathbf{b}$ of the function $F$ at the point $z^{0}$, i.e., $N_{\mathbf{b}}\left(z^{0}, L, F\right)$ is the least number $m_{0}$, for which inequality (1.1) holds for $z=z^{0}$. The maximum in right-hand 
side (1.1) is attained at $m_{0}$. But $0 \leq N_{0} \leq N$. For given $z^{0} \in \mathbb{C}^{n}$ the function $F\left(z^{0}+t \mathbf{b}\right)$ can be developed in power series by powers $t$

$$
F\left(z^{0}+t \mathbf{b}\right)=\sum_{m=0}^{\infty} b_{m}\left(z^{0}\right) t^{m}, b_{m}\left(z^{0}\right)=\frac{\partial_{\mathbf{b}}^{m} F\left(z^{0}\right)}{m !} .
$$

Put $a_{m}\left(z^{0}\right)=\frac{\left|b_{m}\left(z^{0}\right)\right|}{L^{m}\left(z^{0}\right)}=\frac{\left|\partial_{b}^{m} F\left(z^{0}\right)\right|}{m ! L^{m}\left(z^{0}\right)}$. For every $m \in \mathbb{Z}_{+}$the inequality $a_{N_{0}}\left(z^{0}\right) \geq a_{m}\left(z^{0}\right)=R_{0} a_{m}\left(z^{0}\right)$ is true. Then there exists the least number $n_{0} \in\left\{0,1, \ldots, N_{0}\right\}$ such that for all $m \in \mathbb{Z}_{+} a_{n_{0}}\left(z^{0}\right) \geq a_{m}\left(z^{0}\right) R_{N_{0}-n_{0}}$. Thus, $a_{n_{0}}\left(z^{0}\right) \geq a_{N_{0}}\left(z^{0}\right) R_{N_{0}-n_{0}}$ and $a_{j}\left(z^{0}\right)<a_{N_{0}}\left(z^{0}\right) R_{N_{0}-j}$ for $j<n_{0}$, because if $a_{j_{0}}\left(z^{0}\right) \geq a_{N_{0}}\left(z^{0}\right) R_{N_{0}-j_{0}}$ for some $j_{0}<n_{0}$ then $a_{j_{0}}\left(z^{0}\right) \geq$ $a_{m}\left(z^{0}\right) R_{N_{0}-j_{0}}$ for all $m \in \mathbb{Z}_{+}$, but it contradicts the choice of $n_{0}$. From inequalities $a_{j}\left(z^{0}\right)<a_{N_{0}}\left(z^{0}\right) R_{N_{0}-j}\left(j<n_{0}\right)$ and $a_{m}\left(z^{0}\right) \leq a_{N_{0}}\left(z^{0}\right)$ $\left(m>n_{0}\right)$ for $t \in S_{z^{0}}$ and $|t|=\frac{1}{L\left(z^{0}\right)} r_{N_{0}-n_{0}}$ we deduce

$$
\begin{gathered}
\left|F\left(z^{0}+t \mathbf{b}\right)\right|= \\
=\left|b_{n_{0}}\left(z^{0}\right) t^{n_{0}}+\sum_{m \neq n_{0}} b_{m}\left(z^{0}\right) t^{m}\right| \geq\left|b_{n_{0}}\left(z^{0}\right)\right||t|^{n_{0}}-\left.\sum_{m \neq n_{0}}\left|b_{m}\left(z^{0}\right)\right| t\right|^{m} \\
=a_{n_{0}}\left(z^{0}\right) r_{N_{0}-n_{0}}^{n_{0}}-\sum_{m \neq 0} a_{m}\left(z^{0}\right) r_{N_{0}-n_{0}}^{m}=a_{n_{0}}\left(z^{0}\right) r_{N_{0}-n_{0}}^{n_{0}}-\sum_{j<n_{0}} a_{j}\left(z^{0}\right) r_{N_{0}-n_{0}}^{j} \\
-\sum_{m>n_{0}} a_{m}\left(z^{0}\right) r_{N_{0}-n_{0}}^{m} \geq a_{N_{0}}\left(z^{0}\right) R_{N_{0}-n_{0}} r_{N_{0}-n_{0}}^{n_{0}}-\sum_{j<n_{0}} a_{N_{0}}\left(z^{0}\right) R_{N_{0}-j} r_{N_{0}-n_{0}}^{j} \\
-\sum_{m>n_{0}} a_{N_{0}}\left(z^{0}\right) r_{N_{0}-n_{0}}^{m} \geq a_{N_{0}}\left(z^{0}\right) R_{N_{0}-n_{0}} r_{N_{0}-n_{0}}^{n_{0}}-n_{0} a_{N_{0}}\left(z^{0}\right) R_{N_{0}-n_{0}+1} \\
-a_{N_{0}}\left(z^{0}\right) r_{N_{0}-n_{0}}^{n_{0}+1} \frac{1}{1-r_{N_{0}-n_{0}}}=a_{N_{0}}\left(z^{0}\right)\left(R_{N_{0}-n_{0}} r_{N_{0}-n_{0}}^{n_{0}}-\frac{n_{0}}{4 N} R_{N_{0}-n_{0}} r_{N_{0}-n_{0}}^{N}\right. \\
\left.-r_{N_{0}-n_{0}}^{n_{0}} \frac{r_{N_{0}-n_{0}}}{1-r_{N_{0}-n_{0}}}\right) \geq a_{N_{0}}\left(z^{0}\right)\left(R_{N_{0}-n_{0}} r_{N_{0}-n_{0}}^{n_{0}}-\frac{1}{4} R_{N_{0}-n_{0}} r_{N_{0}-n_{0}}^{n_{0}}\right. \\
\left.-\frac{1}{4} R_{N_{0}-n_{0}} r_{N_{0}-n_{0}}^{n_{0}}\right)=\frac{1}{2} a_{N_{0}}\left(z^{0}\right) R_{N_{0}-n_{0}} r_{N_{0}-n_{0}}^{n_{0}} .
\end{gathered}
$$

Besides, for $t \in \mathbb{C}$ we have

$$
\begin{array}{r}
\left|F\left(z^{0}+t \mathbf{b}\right)\right| \leq \sum_{m=0}^{+\infty}\left|b_{m}\left(z^{0}\right)\right||t|^{m}=\sum_{m=0}^{\infty} a_{m}\left(z^{0}\right) r_{N_{0}-n_{0}}^{m} \\
\leq a_{N_{0}}\left(z^{0}\right) \sum_{m=0}^{+\infty} r_{N_{0}-n_{0}}^{m}=\frac{a_{N_{0}}\left(z^{0}\right)}{1-r_{N_{0}-n_{0}}} \leq \frac{a_{N_{0}}\left(z^{0}\right)}{1-1 / 8}=\frac{8}{7} a_{N_{0}}\left(z^{0}\right) .
\end{array}
$$


From (3.9) and (3.10) it follows

$$
\begin{aligned}
& \max \left\{\left|F\left(z^{0}+t \mathbf{b}\right)\right|:|t|=r_{N_{0}-n_{0}} / L\left(z^{0}\right)\right\} \leq \frac{8}{7} a_{N_{0}}\left(z^{0}\right) \\
\leq & \frac{16}{7} \frac{1}{R_{N_{0}-n_{0}}} r_{N_{0}-n_{0}}^{-n_{0}} \min \left\{\left|F\left(z^{0}+t \mathbf{b}\right)\right|:|t|=\frac{r_{N_{0}-n_{0}}}{L\left(z^{0}\right)}\right\} \\
\leq & \frac{16}{7} \frac{1}{R_{N}} r_{N}^{-N} \min \left\{\left|F\left(z^{0}+t \mathbf{b}\right)\right|:|t|=r_{N_{0}-n_{0}} / L\left(z^{0}\right)\right\}
\end{aligned}
$$

i.e., (3.8) holds with $P_{2}(R)=\frac{16}{7 R_{N} r_{N}^{N}}, \eta(R)=r_{N}=\frac{1}{8 R_{N}}$ and $r=$ $r_{N_{0}-n_{0}}$. Theorem is proved.

Below we will prove the sufficint conditions which are symmetric to necessary conditions from Theorem 3.2

Theorem 3.3. Let $L \in Q_{\mathrm{b}}^{n}, F \in \widetilde{\mathcal{H}}_{\mathrm{b}}^{n}$. If there exist $R>0, P_{2} \geq 1$ and $\eta \in(0, R)$ such that for all $z^{0} \in \mathbb{C}^{n}$ and some $r=r\left(z^{0}\right) \in[\eta, R]$ inequality (3.8) is valid, then the function $F$ has bounded L-index in the direction $\mathbf{b}$.

Proof. Directly this proposition was unknown for entire functions of bounded index, i.e. for functions of one variable. Firstly, it was obtained for entire functions of bounded $L$-index in direction in $[10,11]$. In view of Proposition 3.1 it is sufficient to show that there exists $P_{1}$ such that for all $z^{0} \in \mathbb{C}^{n}$

$$
\begin{aligned}
& \max \left\{\left|F\left(z^{0}+t \mathbf{b}\right)\right|:|t|=(R+1) / L\left(z^{0}\right)\right\} \\
& \leq P_{1} \max \left\{\left|F\left(z^{0}+t \mathbf{b}\right)\right|:|t|=R / L\left(z^{0}\right)\right\} .
\end{aligned}
$$

Suppose that there exist $R>0, P_{2} \geq 1$ and $\eta \in(0, R)$ such that for all $z^{0} \in \mathbb{C}^{n}$ and some $r=r\left(z^{0}\right) \in[\eta, R]$ one has

$$
\max \left\{\left|F\left(z^{0}+t \mathbf{b}\right)\right|:|t|=r / L\left(z^{0}\right)\right\} \leq P_{2} \min \left\{\left|F\left(z^{0}+t \mathbf{b}\right)\right|:|t|=r / L\left(z^{0}\right)\right\} .
$$

Denote $L^{*}=\max \left\{L\left(z^{0}+t \mathbf{b}\right):|t| \leq(2 R+2) / L\left(z^{0}\right)\right\}, \rho_{0}=R / L\left(z^{0}\right)$, $\rho_{k}=\rho_{0}+k \eta / L^{*}, k \in \mathbb{Z}_{+}$. We have

$$
\frac{\eta}{L^{*}}<\frac{R}{L^{*}} \leq \frac{R}{L\left(z^{0}\right)}<\frac{2 R+2}{L\left(z^{0}\right)}-\frac{R+1}{L\left(z^{0}\right)}
$$

Then there exists $n^{*} \in \mathbb{N}$, independent of $z^{0}$ such that

$$
\rho_{p-1}<\frac{R+1}{L\left(z^{0}\right)} \leq \rho_{p} \leq \frac{2 R+2}{L\left(z^{0}\right)}
$$


for some $p=p\left(z^{0}\right) \leq n^{*}$, because $L \in Q_{\mathbf{b}}^{n}$. Indeed,

$$
\begin{gathered}
\left(\frac{2 R+2}{L\left(z^{0}\right)}-\rho_{0}\right) /\left(\frac{\eta}{L^{*}}\right)=\frac{(R+2) L^{*}}{\eta L\left(z^{0}\right)} \\
=\frac{R+2}{\eta} \max \left\{\frac{L\left(z^{0}+t \mathbf{b}\right)}{L\left(z^{0}\right)}:|t| \leq \frac{2 R+2}{L\left(z^{0}\right)}\right\} \leq \frac{R+2}{\eta} \lambda_{\mathbf{b}}(2 R+2) .
\end{gathered}
$$

Therefore, $n^{*}=\left[\frac{R+2}{\eta} \lambda_{\mathbf{b}}(2 R+2)\right]$, where $[a]$ is integer part of number $a \in \mathbb{R}$. Let $\left|F\left(z^{0}+t_{k}^{* *} \mathbf{b}\right)\right|=\max \left\{\left|F\left(z^{0}+t \mathbf{b}\right)\right|: t \in c_{k}\right\}, c_{k}=\{t \in \mathbb{C}:$ $\left.|t|=\rho_{k}\right\}$, and $t_{k}^{*}$ be an intersection point of the segment $\left[0, t_{k}^{* *}\right]$ with the circle $c_{k-1}$. Then for every $r>\eta$ and for each $k \leq n^{*}$ the inequality holds $\left|t_{k}^{* *}-t_{k}^{*}\right|=\frac{\eta}{L^{*}} \leq \frac{r}{L\left(z^{0}+t_{k}^{*} \mathbf{b}\right)}$. Thus, for some $r=r\left(z^{0}+t_{k}^{*} \mathbf{b}\right) \in[\eta, R]$ we deduce

$$
\begin{gathered}
\left|F\left(z^{0}+t_{k}^{* *} \mathbf{b}\right)\right| \leq \max \left\{\left|F\left(z^{0}+t \mathbf{b}\right)\right|:\left|t-t_{k}^{*}\right|=r / L\left(z^{0}+t_{k}^{*} \mathbf{b}\right)\right\} \\
\leq P_{2} \min \left\{\left|F\left(z^{0}+t \mathbf{b}\right)\right|:\left|t-t_{k}^{*}\right|=r / L\left(z^{0}+t_{k}^{*} \mathbf{b}\right)\right\} \\
\leq P_{2} \min \left\{\left|F\left(z^{0}+t \mathbf{b}\right)\right|:\left|t-t_{k}^{*}\right|=r / L\left(z^{0}+t_{k}^{*} \mathbf{b}\right),\left|t-t_{0}\right| \leq \rho_{k-1}\right\} \\
\leq P_{2} \max \left\{\left|F\left(z^{0}+t \mathbf{b}\right)\right|: t \in c_{k-1}\right\} .
\end{gathered}
$$

Hence,

$$
\begin{gathered}
\max \left\{\left|F\left(z^{0}+t \mathbf{b}\right)\right|:|t|=(R+1) / L\left(z^{0}\right)\right\} \\
\leq \max \left\{\left|F\left(z^{0}+t \mathbf{b}\right)\right|: t \in c_{p}\right\} \leq P_{2} \max \left\{\left|F\left(z^{0}+t \mathbf{b}\right)\right|: t \in c_{p-1}\right\} \\
\leq \ldots \leq\left(P_{2}\right)^{p} \max \left\{\left|F\left(z^{0}+t \mathbf{b}\right)\right|: t \in c_{0}\right\} \\
\leq\left(P_{2}\right)^{n^{*}} \max \left\{\left|F\left(z^{0}+t \mathbf{b}\right)\right|:|t|=R / L\left(z^{0}\right)\right\} .
\end{gathered}
$$

We obtained (3.11) with $P_{1}=\left(P_{2}\right)^{n^{*}}$. Theorem 3.3 is proved.

\section{Estimate of directional logarithmic derivative}

In this section we deduce analog of logarithmic criterion for function from the class $\widetilde{\mathcal{H}}_{\mathrm{b}}^{n}$. The one-dimensional analog of the criterion is efficient to investigate boundedness of $l$-index of infinite products $[12,28,30]$. As necessary conditions the criterion was obtained by G. H. Fricke $[14,15]$ for entire functions of bounded index.

Below we prove the criterion of $L$-index boundedness in direction, which describes behavior of directional logarithmic derivative and distribution of zeros. We need additional denotations.

Denote

$$
G_{r}(F):=G_{r}^{\mathbf{b}}(F):=\bigcup_{z: F(z)=0}\{z+t \mathbf{b}:|t|<r / L(z)\},
$$


where $a_{k}^{0}$ are zeros of the function $F\left(z^{0}+t \mathbf{b}\right)$ for given $z^{0} \in \mathbb{C}^{n}$.

By $n\left(r, z^{0}, 1 / F\right)=\sum_{\left|a_{k}^{0}\right| \leq r} 1$ we denote counting function of zeros $a_{k}^{0}$.

Theorem 4.1. Let $F \in \widetilde{\mathcal{H}}_{\mathrm{b}}^{n}, L \in Q_{\mathrm{b}}^{n}$. If the function $F$ has bounded $L$-index in the direction $\mathbf{b}$, then

1) for each $r>0$ there exists $P=P(r)>0$ such that for every $z \in \mathbb{C}^{n} \backslash G_{r}^{\mathbf{b}}(F)$

$$
\left|\frac{\partial_{\mathbf{b}} F(z)}{F(z)}\right| \leq P L(z)
$$

2) for any $r>0$ there exists $\tilde{n}(r) \in \mathbb{Z}_{+}$such that for all $z^{0} \in \mathbb{C}^{n}$ such that $F\left(z^{0}+t \mathbf{b}\right) \not \equiv 0$ one has

$$
n\left(\frac{r}{L\left(z^{0}\right)}, z^{0}, \frac{1}{F}\right) \leq \widetilde{n}(r) .
$$

Proof. Out proof is based on the proof of appropriate proposition for entire functions of bounded $L$-index in direction $[6,8]$.

Firstly, we will show that the condition " $F(z)$ is of bounded $L$-index in the direction" implies that for every $z^{0} \in \mathbb{C}^{n} \backslash G_{r}^{\mathbf{b}}(F)(r>0)$ and for each $\widetilde{a}^{k}=z^{0}+a_{k}^{0} \mathbf{b}$ one has

$$
\left|z^{0}-\widetilde{a}_{k}\right|>\frac{r|\mathbf{b}|}{2 L\left(\widetilde{z}^{0}\right) \lambda_{\mathbf{b}}(r)} .
$$

On the contrary, suppose that there exist $z^{0} \in \mathbb{C}^{n} \backslash G_{r}^{\mathbf{b}}(F)$ and $\widetilde{a}^{k}=z^{0}+$ $a_{k}^{0} \mathbf{b}$ such that $\left|z^{0}-\widetilde{a}_{k}\right| \leq \frac{r|\mathbf{b}|}{2 L\left(\widetilde{z}^{0}\right) \lambda_{\mathbf{b}}(r)} \leq \frac{r|\mathbf{b}|}{2 L\left(z^{0}\right)}<\frac{r|\mathbf{b}|}{L\left(z^{0}\right)}$. Hence, $\left|a_{k}^{0}\right|<$ $\frac{r}{L\left(z^{0}\right)}$. But for $\lambda_{2}^{\mathbf{b}}$ the following estimates hold $L\left(\widetilde{a}^{k}\right) \leq \lambda_{\mathbf{b}}(r) L\left(z^{0}\right)$. Then $\left|z^{0}-\widetilde{a}^{k}\right|=|\mathbf{b}| \cdot\left|a_{k}^{0}\right| \leq \frac{r|\mathbf{b}|}{2 L\left(\widetilde{a}^{k}\right)}$, i.e., $\left|a_{k}^{0}\right| \leq \frac{r}{2 L\left(\widetilde{a}^{k}\right)}$. This contradicts $\widetilde{z}^{0} \in \mathbb{C}^{n} \backslash G_{r}^{\mathbf{b}}(F)$.

Put in Theorem $3.2 R=\frac{r}{2 \lambda_{\mathbf{b}}(r)}$. Then there exist $P_{2} \geq 1$ and $\eta \in$ $(0, R)$ such that for every $\widetilde{z^{0}}=z^{0} \in \mathbb{C}^{n}$ and for some $r^{*} \in[\eta, R]$ inequality (3.8) holds with $r^{*}$ instead $r$. Therefore, by Cauchy's inequality

$$
\begin{gathered}
\left|\partial_{\mathbf{b}} F\left(z^{0}\right)\right| \leq \frac{L\left(z^{0}\right)}{r^{*}} \max \left\{\left|F\left(z^{0}+t \mathbf{b}\right):\right| t \mid=r^{*} / L\left(z^{0}\right)\right\} \\
\leq P_{2} \frac{L\left(z^{0}\right)}{\eta} \min \left\{\left|F\left(z^{0}+t \mathbf{b}\right)\right|:|t|=r^{*} / L\left(z^{0}\right)\right\} .
\end{gathered}
$$


In view of (4.4) for every point $z^{0}+t_{0} \mathbf{b} \in \mathbb{C}^{n} \backslash G_{r}^{\mathbf{b}}(F)$ the following set $\left\{z^{0}+t \mathbf{b}:|t| \leq \frac{r}{2 \lambda_{2}^{\mathbf{b}}(r) L\left(z^{0}\right)}\right\}$ does not contain zeros of the function $F\left(z^{0}+t \mathbf{b}\right)$. Therefore, applying the maximum modulus principle to $1 / F$ as a function of variable $t$, we have

$$
\left|F\left(z^{0}\right)\right| \geq \min \left\{\left|F\left(z^{0}+t \mathbf{b}\right)\right|:|t|=r^{*} / L\left(z^{0}\right)\right\}
$$

From inequalities (4.5) and (4.6) it follows (4.2) with $P=\frac{P_{2}}{\eta}$.

Now we will prove that for a function $F$ of bounded $L$-index in the direction $\mathbf{b}$ there exists $P_{3}>0$ such that for every $z^{0} \in \mathbb{C}^{n}\left(F\left(z^{0}+t \mathbf{b}\right) \not \equiv\right.$ $0), r \in(0,1]$

$$
\begin{gathered}
n\left(r / L\left(z^{0}\right), z^{0}, 1 / F\right) \min \left\{\left|F\left(z^{0}+t \mathbf{b}\right)\right|:|t|=r / L\left(z^{0}\right)\right\} \\
\leq P_{3} \max \left\{\left|F\left(z^{0}+t \mathbf{b}\right)\right|:|t|=1 / L\left(z^{0}\right)\right\} .
\end{gathered}
$$

Applying Cauchy's inequality and Theorem 3.1 for all $t$ on the circle $|t|=\frac{1}{L\left(z^{0}\right)}$, we have

$$
\begin{gathered}
\left|\partial_{\mathbf{b}} F\left(z^{0}+t \mathbf{b}\right)\right| \leq \frac{L\left(z^{0}\right)}{r} \max \left\{\left|F\left(z^{0}+\theta \mathbf{b}\right)\right|:|\theta-t|=\frac{r}{L\left(z^{0}\right)}\right\} \\
\leq \frac{L\left(z^{0}\right)}{r} \max \left\{\left|F\left(z^{0}+t \mathbf{b}\right)\right|:|t|=\frac{r+1}{L\left(z^{0}\right)}\right\} \\
\leq \frac{P_{1}(1, r+1)}{r} L\left(z^{0}\right) \max \left\{\left|F\left(z^{0}+t \mathbf{b}\right)\right|:|t|=\frac{1}{L\left(z^{0}\right)}\right\} .
\end{gathered}
$$

If $F\left(z^{0}+t \mathbf{b}\right) \neq 0$ on the circle $\left\{t \in \mathbb{C}:|t|=r / L\left(z^{0}\right)\right\}$, then

$$
\begin{aligned}
& n\left(\frac{r}{L\left(z^{0}\right)}, z^{0}, \frac{1}{F}\right)=\left|\frac{1}{2 \pi i} \int_{|t|=\frac{r}{L\left(z^{0}\right)}} \frac{\partial_{\mathbf{b}} F\left(z^{0}+t \mathbf{b}\right)}{F\left(z^{0}+t \mathbf{b}\right)} d t\right| \\
& \leq \frac{\max \left\{\left|\partial_{\mathbf{b}} F\left(z^{0}+t \mathbf{b}\right)\right|:|t|=r / L\left(z^{0}\right)\right\}}{\min \left\{\left|F\left(z^{0}+t \mathbf{b}\right)\right|:|t|=r / L\left(z^{0}\right)\right\}} \frac{r}{L\left(z^{0}\right)} .
\end{aligned}
$$

From (4.8) and (4.9) we deduce

$$
\begin{gathered}
n\left(r / L\left(z^{0}\right), z^{0}, 1 / F\right) \min \left\{\left|F\left(z^{0}+t \mathbf{b}\right)\right|:|t|=r / L\left(z^{0}\right)\right\} \\
\quad \leq \frac{r}{L\left(z^{0}\right)} \max \left\{\left|\partial_{\mathbf{b}} F\left(z^{0}+t \mathbf{b}\right)\right|:|t|=r / L\left(z^{0}\right)\right\} \\
\quad \leq \frac{1}{L\left(z^{0}\right)} \max \left\{\left|\partial_{\mathbf{b}} F\left(z^{0}+t \mathbf{b}\right)\right|:|t|=1 / L\left(z^{0}\right)\right\} \\
\leq P_{1}(1, r+1) /(r) \max \left\{\left|F\left(z^{0}+t \mathbf{b}\right)\right|:|t|=1 / L\left(z^{0}\right)\right\} .
\end{gathered}
$$


Thus, we obtained (4.7) with $P_{3}=\frac{P_{1}(1, r+1)}{r}$. If the function $F\left(z^{0}+t \mathbf{b}\right)$ has zeros on the circle $\left\{t \in \mathbb{C}:|t|=r / L\left(z^{0}\right)\right\}$, then inequality (4.7) is obvious.

Set $R=1$ in Theorem 3.2. Then there exist $P_{2}=P_{2}(1) \geq 1$ and $\eta \in(0,1)$ such that for every $z^{0} \in \mathbb{C}^{n}$ and some $r^{*}=r^{*}\left(z^{0}, t_{0}\right) \in[\eta, 1]$

$$
\max \left\{\left|F\left(z^{0}+t \mathbf{b}\right)\right|:|t|=\frac{r^{*}}{L\left(z^{0}\right)}\right\} \leq P_{2} \min \left\{\left|F\left(z^{0}+t \mathbf{b}\right)\right|:|t|=\frac{r^{*}}{L\left(z^{0}\right)}\right\} .
$$

Next, by Theorem 3.1 there exists $P_{1} \geq 1$ such that for all $z^{0} \in \mathbb{B}^{n}$

$$
\begin{gathered}
\max \left\{\left|F\left(z^{0}+t \mathbf{b}\right)\right|:|t|=1 / L\left(z^{0}\right)\right\} \\
\leq P_{1}(1, \eta) \max \left\{\left|F\left(z^{0}+t \mathbf{b}\right)\right|:|t|=\eta / L\left(z^{0}\right)\right\} \\
\leq P_{1}(1, \eta) \max \left\{\left|F\left(z^{0}+t \mathbf{b}\right)\right|:|t|=r^{*} / L\left(z^{0}\right)\right\} \\
\leq P_{1}(1, \eta) P_{2} \min \left\{\left|F\left(z^{0}+t \mathbf{b}\right)\right|:|t|=r^{*} / L\left(z^{0}\right)\right\} .
\end{gathered}
$$

Taking into account (4.7), we obtain

$$
\begin{gathered}
n\left(r^{*} / L\left(z^{0}\right), z^{0}, 1 / F\right) \min \left\{\left|F\left(z^{0}+t \mathbf{b}\right)\right|:|t|=r^{*} / L\left(z^{0}\right)\right\} \\
\quad \leq P_{3} P_{1}(1, \eta) P_{2} \min \left\{\left|F\left(z^{0}+t \mathbf{b}\right)\right|:|t|=r^{*} / L\left(z^{0}\right)\right\}
\end{gathered}
$$

i.e., $n\left(\frac{r^{*}}{L\left(z^{0}\right)}, z^{0}, \frac{1}{F}\right) \leq P_{1}(1, \eta) P_{2} P_{3}$. Hence,

$$
n\left(\frac{r^{*}}{L\left(z^{0}\right)}, z^{0}, \frac{1}{F}\right) \leq P_{4}=P_{1}(1, \eta) P_{2} P_{3}=\frac{P_{1}(1, \eta) P_{2}(1) P_{1}(1, r+1)}{r}
$$

If $r \in(0, \eta]$, then property (4.3) is proved.

Let $r>\eta$ and $L^{*}=\max \left\{L\left(z^{0}+t \mathbf{b}\right):|t|=\frac{r}{L\left(z^{0}\right)}\right\}$. Using properties $Q_{\mathbf{b}}^{n}$, we have $L^{*} \leq \lambda_{\mathbf{b}}(r) L\left(z^{0}\right)$. Put $\rho=\frac{\eta}{L\left(z^{0}\right) \lambda_{\mathbf{b}}(r)}, R=\frac{r}{L\left(z^{0}\right)}$. We can cover every set $\bar{K}=\left\{z^{0}+t \mathbf{b}:|t| \leq R\right\}$ by a finite number $m=m(r)$ of closed sets $\bar{K}_{j}=\left\{z^{0}+t \mathbf{b}:\left|t-t_{j}\right| \leq \rho\right\}$, where $t_{j} \in \bar{K}$. Since $\frac{\eta}{\lambda_{\mathbf{b}}(r) L\left(z^{0}\right)} \leq \frac{\eta}{L^{*}} \leq \frac{\eta}{L\left(z^{0}+t_{j} \mathbf{b}\right)}$ every set $\bar{K}_{j}$ contains at least $\left[P_{4}\right]$ zeros of the function $F\left(z^{0}+t \mathbf{b}\right)$. Therefore, $n\left(\frac{r}{L\left(z^{0}\right)}, z^{0}, 1 / F\right) \leq \widetilde{n}(r)=\left[P_{4}\right] m(r)$ and property (4.3) is proved.

By $n_{z^{0}}(r, F)=n_{\mathbf{b}}\left(r, z^{0}, 1 / F\right):=\sum_{\left|a_{k}^{0}\right| \leq r} 1$ we denote counting function of zeros $a_{k}^{0}$ for the slice function $F\left(z^{0}+t \mathbf{b}\right)$ in the disc $\{t \in \mathbb{C}:|t| \leq$ $r\}$. If for given $z^{0} \in \mathbb{C}^{n}$ and for all $t \in \mathbb{C} F\left(z^{0}+t \mathbf{b}\right) \equiv 0$, then we put $n_{z^{0}}(r)=-1$. Denote $n(r)=\sup _{z \in \mathbb{C}^{n}} n_{z}(r / L(z))$. 
Theorem 4.2. Let $L \in Q_{\mathrm{b}}^{n}, F \in \widetilde{\mathcal{H}}_{\mathrm{b}}^{n}$. If the following conditions are satisfied

1) there exists $r_{1}>0$ such that $n\left(r_{1}\right) \in[-1 ; \infty)$;

2) there exist $r_{2} \in>0, P>0$ such that $2 r_{2} \cdot n\left(r_{1}\right)<r_{1} / \lambda_{\mathbf{b}}\left(r_{1}\right)$ and for all $z \in \mathbb{C}^{n} \backslash G_{r_{2}}(F)$ inequality (4.2) is true;

then the function $F$ has bounded $L$-index in the direction $\mathbf{b}$.

Proof. Analog of the proposition was firstly deduced for entire functions of bounded $L$-index in direction $[10,11]$. Suppose that conditions 1) and 2) are true.

At first, we consider the case $n\left(r_{1}\right) \in\{-1 ; 0\}$. Then in the best case the function $F$ can only identically equals zero on the complex line $z^{*}+t \mathbf{b}$ for some $z^{*} \in \mathbb{C}^{n}$, i.e., $F\left(z^{*}+t \mathbf{b}\right) \equiv 0$. For all points lying on such complex lines inequality (3.8) is obvious.

Let $z^{0} \in \mathbb{C}^{n} \backslash G_{r_{2}}$. For any points $t_{1}$ and $t_{2}$ such that $\left|t_{j}\right|=\frac{r_{2}}{L\left(z_{0}\right)}$, $j \in\{1,2\}$, one has

$$
\begin{gathered}
\ln \left|\frac{F\left(z^{0}+t_{2} \mathbf{b}\right)}{F\left(z^{0}+t_{1} \mathbf{b}\right)}\right| \leq \int_{t_{1}}^{t_{2}}\left|\frac{\partial_{\mathbf{b}} F\left(z^{0}+t \mathbf{b}\right)}{F\left(z^{0}+t \mathbf{b}\right)}\right||d t| \\
\leq P \int_{t_{1}}^{t_{2}} L\left(z^{0}+t \mathbf{b}\right)|d t| \leq P \lambda_{\mathbf{b}}\left(r_{2}\right) L\left(z^{0}\right) \frac{\pi r_{2}}{L\left(z^{0}\right)} \leq \pi r_{2} P \lambda_{\mathbf{b}}\left(r_{2}\right)
\end{gathered}
$$

(we also use that $L \in Q_{\mathrm{b}}^{n}$ ). Hence,

$$
\max \left\{\left|F\left(z^{0}+t \mathbf{b}\right)\right|:|t|=\frac{r_{2}}{L\left(z^{0}\right)}\right\} \leq P_{2} \min \left\{\left|F\left(z^{0}+t \mathbf{b}\right)\right|:|t|=\frac{r_{1}}{L\left(z^{0}\right)}\right\},
$$

where $P_{2}=\exp \left\{\pi r_{2} P \lambda_{2}\left(r_{2}\right)\right\}$. Therefore, by Theorem 3.3 the function $F$ has bounded $L$-index in the direction $\mathbf{b}$.

Let $r_{1}>0$ be a such that $n\left(r_{1}\right) \in[1 ; \infty)$ and $2 n\left(r_{1}\right) r_{2}<r_{1} / \lambda_{\mathbf{b}}\left(r_{1}\right)$. Put $c=\frac{r_{1}}{2 r_{2} \lambda_{\mathbf{b}}\left(r_{1}\right)}-n\left(r_{1}\right)>0$. Clearly, $r_{2}=r_{1} /\left(2\left(n\left(r_{1}\right)+c\right) \lambda_{\mathbf{b}}\left(r_{1}\right)\right)$.

Under condition 1) each set $\bar{K}=\left\{z^{0}+t \mathbf{b}:|t| \leq \frac{r_{1}}{L\left(z^{0}\right)}\right\}$ has no more $n\left(r_{1}\right)$ zeros of the function $F$, where $F\left(z^{0}+t \mathbf{b}\right) \not \equiv 0$.

Under condition 2) there exists $P>0$ such that $\left|\frac{\partial_{\mathbf{b}} F(z)}{F(z)}\right| \leq P L(z)$ for every $z \in \mathbb{C}^{n} \backslash G_{r_{2}}$, i.e., for all $z \in \bar{K}$, lying outside the sets

$$
\left\{z^{0}+t \mathbf{b}:\left|t-a_{k}^{0}\right|<\frac{r_{2}}{L\left(z^{0}+a_{k}^{0} \mathbf{b}\right)}\right\}
$$

where $a_{k}^{0} \in \bar{K}$ are zeros of the slice function $F\left(z^{0}+t \mathbf{b}\right) \not \equiv 0$. By definition $\lambda_{\mathbf{b}}$ we obtain

$$
L\left(z^{0}\right) / \lambda_{\mathbf{b}}\left(r_{1}\right) \leq L\left(z^{0}+a_{k}^{0} \mathbf{b}\right) .
$$


Then $\left|\frac{\partial_{\mathbf{b}} F(z)}{F(z)}\right| \leq P L(z)$ for every point $z \in \mathbb{C}^{n}$, lying outside union of the sets

$$
c_{k}^{0}=\left\{z^{0}+t \mathbf{b}:\left|t-a_{k}^{0}\right| \leq \frac{r_{2} \lambda_{\mathbf{b}}\left(r_{1}\right)}{L\left(z^{0}\right)}=\frac{r_{1}}{2\left(n\left(r_{1}\right)+c\right) L\left(z^{0}\right)}\right\} .
$$

The total sum of diameters of the sets $c_{k}^{0}$ does not exceed the value $\frac{r_{1} n\left(r_{1}\right)}{\left(n\left(r_{1}\right)+c\right) L\left(z^{0}\right)}<\frac{r_{1}}{L\left(z^{0}\right)}$. Hence, there exists a set $\widetilde{c}^{0}=\left\{z^{0}+t \mathbf{b}:|t|=\frac{r}{L\left(z^{0}\right)}\right\}$, where $\frac{r_{1} \min \{1, c\}}{2\left(n\left(r_{1}\right)+c\right)}=\eta<r<r_{1}$, such that, for all $z \in \widetilde{c}^{0}$

$$
\left|\frac{\partial_{\mathbf{b}} F(z)}{F(z)}\right| \leq P L(z) \leq P \lambda_{\mathbf{b}}(r) L\left(z^{0}\right) \leq P \lambda_{\mathbf{b}}\left(r_{1}\right) L\left(z^{0}\right) .
$$

For any points $z_{1}=z^{0}+t_{1} \mathbf{b}$ and $z_{2}=z^{0}+t_{2} \mathbf{b}$ with $\widetilde{c}^{0}$ one has

$$
\begin{aligned}
& \ln \left|\frac{F\left(z^{0}+t_{2} \mathbf{b}\right)}{F\left(z^{0}+t_{1} \mathbf{b}\right)}\right| \leq \int_{t_{1}}^{t_{2}}\left|\frac{\partial_{\mathbf{b}} F\left(z^{0}+t \mathbf{b}\right)}{F\left(z^{0}+t \mathbf{b}\right)}\right||d t| \\
& \leq P \lambda_{2}\left(r_{1}\right) L\left(z^{0}\right) \frac{\pi r}{L\left(z^{0}\right)} \leq \pi r_{1} P\left(r_{2}\right) \lambda_{\mathbf{b}}\left(r_{1}\right) .
\end{aligned}
$$

Therefore,

$\max \left\{\left|F\left(z^{0}+t \mathbf{b}\right)\right|:|t|=\frac{r}{L\left(z^{0}\right)}\right\} \leq P_{2} \min \left\{\left|F\left(z^{0}+t \mathbf{b}\right)\right|:|t|=\frac{r}{L\left(z^{0}\right)}\right\}$,

where $P_{2}=\exp \left\{\pi r_{1} P\left(r_{2}\right) \lambda_{\mathbf{b}}\left(r_{1}\right)\right\}$. If $F\left(z^{0}+t \mathbf{b}\right) \equiv 0$, then inequality (4.10) is obvious. By Theorem 3.3 the function $F(z)$ has bounded $L$ index in the direction $\mathbf{b}$. Theorem 4.2 is proved.

\section{Analog of Hayman's Theorem}

Below we formulate and prove criterion which is analog of Hayman's Theorem [18].

Theorem 5.1. Let $L \in Q_{\mathrm{b}}^{n}$. A function $F \in \widetilde{\mathcal{H}}_{\mathrm{b}}^{n}$ is of bounded L-index in the direction $\mathbf{b}$ if and only if there exist $p \in \mathbb{Z}_{+}$and $C>0$ such that for every $z \in \mathbb{C}^{n}$ one has

$$
\frac{\left|\partial_{\mathbf{b}}^{p+1} F(z)\right|}{L^{p+1}(z)} \leq C \max \left\{\frac{\left|\partial_{\mathbf{b}}^{k} F(z)\right|}{L^{k}(z)}: 0 \leq k \leq p\right\} .
$$


Proof. The proof use ideas from the proof for entire functions of bounded $L$-index in direction $[6,8]$. Also there are known analogs of Hayman's theorem for other classes of analytic functions $[4,9]$.

Necessity. If $N_{\mathbf{b}}(F, L)<+\infty$, then by definition of boundedness of $L$-index in direction we obtain (5.1) with $p=N_{\mathbf{b}}(F, L)$ and $C=$ $\left(N_{\mathbf{b}}(F, L)+1\right)$ !

Sufficiency. Let inequality (5.1) be fulfilled, $z^{0} \in \mathbb{C}^{n}$ and $K=$ $\left\{t \in \mathbb{C}:|t| \leq 1 / L\left(z^{0}\right)\right\}$. Since $L \in Q_{\mathbf{b}}^{n}$, for every $t \in K$ from (5.1) it follows

$$
\begin{gathered}
\frac{\left|\partial_{\mathbf{b}}^{p+1} F\left(z^{0}+t \mathbf{b}\right)\right|}{L^{p+1}\left(z^{0}\right)} \leq\left(\frac{L\left(z^{0}+t \mathbf{b}\right)}{L\left(z^{0}\right)}\right)^{p+1} \frac{\left|\partial_{\mathbf{b}}^{p+1} F\left(z^{0}+t \mathbf{b}\right)\right|}{L^{p+1}\left(z^{0}+t \mathbf{b}\right)} \\
\leq\left(\lambda_{\mathbf{b}}(1)\right)^{p+1} \frac{\left|\partial_{\mathbf{b}}^{p+1} F\left(z^{0}+t \mathbf{b}\right)\right|}{L^{p+1}\left(z^{0}+t \mathbf{b}\right)} \leq C\left(\lambda_{\mathbf{b}}(1)\right)^{p+1} \max _{0 \leq k \leq p}\left\{\frac{\left|\partial_{\mathbf{b}}^{k} F\left(z^{0}+t \mathbf{b}\right)\right|}{L^{k}\left(z^{0}+t \mathbf{b}\right)}\right\} \\
\leq C\left(\lambda_{\mathbf{b}}(1)\right)^{p+1} \max \left\{\left(\frac{L\left(z^{0}\right)}{L\left(z^{0}+t \mathbf{b}\right)}\right)^{k} \frac{\left|\partial_{\mathbf{b}}^{k} F\left(z^{0}+t \mathbf{b}\right)\right|}{L^{k}\left(z^{0}\right)}: 0 \leq k \leq p\right\} \\
\leq C\left(\lambda_{\mathbf{b}}(1)\right)^{p+1} \max \left\{\frac{\left|\partial_{\mathbf{b}}^{k} F\left(z^{0}+t \mathbf{b}\right)\right|}{L^{k}\left(z^{0}\right)}\left(\lambda_{\mathbf{b}}(1)\right)^{k}: 0 \leq k \leq p\right\} \leq B g_{z^{0}}(t),
\end{gathered}
$$

where $B=C\left(\lambda_{\mathbf{b}}(1)\right)^{2 p+1}$ and $g_{z^{0}}(t)=\max \left\{\frac{\left|\partial_{\mathbf{b}}^{k} F\left(z^{0}+t \mathbf{b}\right)\right|}{L^{k}\left(z^{0}\right)}: 0 \leq k \leq p\right\}$.

Let us denote $\gamma_{1}=\left\{t \in \mathbb{C}:|t|=\frac{1}{4 L\left(z^{0}\right)}\right\}, \gamma_{2}=\left\{t \in \mathbb{C}:|t|=\frac{2}{L\left(z^{0}\right)}\right\}$. Choose arbitrarily two points $t_{1} \in \gamma_{1}, t_{2} \in \gamma_{2}$ and connect them by a piecewise analytic curve $\gamma=(t=t(s), 0 \leq s \leq T)$ such that $g_{z^{0}}(t) \neq 0$ for $t \in \gamma$. We construct the curve $\gamma$ such that its length $|\gamma|$ does not exceed $\frac{9}{2 L\left(z^{0}\right)}$.

The function $g_{z^{0}}(t(s))$ is continuous on $[0, T]$. Without loss of generality we may assume that the function $t=t(s)$ is analytic on $[0, T]$. Otherwise, one can consider each interval of analyticity of this function separately and repeat the corresponding considerations, which are given below on $[0, T]$. First, we show that the function $g_{z^{0}}(t(s))$ is continuously differentiable on $[0, T]$ except possibly a finite set of points. For arbitrary $k_{1}, k_{2}, 0 \leq k_{1} \leq k_{2} \leq p$, either $\frac{\left|\partial_{\mathbf{b}}^{k_{1}} F\left(z^{0}+t(s) \mathbf{b}\right)\right|}{L^{k_{1}}\left(z^{0}\right)} \equiv \frac{\left|\partial_{\mathbf{b}}^{k_{2}} F\left(z^{0}+t(s) \mathbf{b}\right)\right|}{L^{k_{2}}\left(z^{0}\right)}$ ot the equality $\frac{\left|\partial_{\mathbf{b}}^{k_{1}} F\left(z^{0}+t(s) \mathbf{b}\right)\right|}{L^{k_{1}}\left(z^{0}\right)}=\frac{\left|\partial_{\mathbf{b}}^{k_{2}} F\left(z^{0}+t(s) \mathbf{b}\right)\right|}{L^{k_{2}}\left(z^{0}\right)}$ is true for a finite set of points $s_{k} \in[0, T]$. Then we can split the segment $[0, T]$ onto a finite number of segments such that on each of them $g_{z^{0}}(t(s)) \equiv \frac{\left|\partial_{\mathbf{b}}^{k} F\left(z^{0}+t(z) \mathbf{b}\right)\right|}{L^{k}\left(z^{0}\right)}$ for some $k, 0 \leq k \leq p$. It means that the function $g_{z^{0}}(t(s))$ is continuously 
differentiable with exception, perhaps, of a finite sets of points. Taking into account (5.2), we obtain

$$
\begin{gathered}
\frac{d g_{z^{0}}(t(s))}{d s} \leq \max \left\{\frac{d}{d s}\left(\frac{\left|\partial_{\mathbf{b}}^{k} F\left(z^{0}+t(s) \mathbf{b}\right)\right|}{L^{k}\left(z^{0}\right)}\right): 0 \leq k \leq p\right\} \\
\leq \max \left\{\left|\partial_{\mathbf{b}}^{k+1} F\left(z^{0}+t(s) \mathbf{b}\right)\right|\left|t^{\prime}(s)\right| / L^{k}\left(z^{0}\right): 0 \leq k \leq p\right\} \\
=L\left(z^{0}\right)\left|t^{\prime}(s)\right| \max \left\{\left|\partial_{\mathbf{b}}^{k+1} F\left(z^{0}+t(s) \mathbf{b}\right)\right| / L^{k+1}\left(z^{0}\right): 0 \leq k \leq p\right\} \\
\leq B g_{z^{0}}(t(s))\left|t^{\prime}(s)\right| L\left(z^{0}\right) .
\end{gathered}
$$

Hence, we have

$$
\left|\ln \frac{g_{z^{0}}\left(t_{2}\right)}{g_{z^{0}}\left(t_{1}\right)}\right|=\left|\int_{0}^{T} \frac{d g_{z^{0}}(t(s))}{g_{z^{0}}(t(s))}\right| \leq B L\left(z^{0}\right) \int_{0}^{T}\left|t^{\prime}(s)\right| d s=B L\left(z^{0}\right)|\gamma| \leq 4.5 B .
$$

If we choose a point $t_{2} \in \gamma_{2}$, such that

$$
\left|F\left(z^{0}+t_{2} \mathbf{b}\right)\right|=\max \left\{\left|F\left(z^{0}+t \mathbf{b}\right)\right|:|t|=2 / L\left(z^{0}\right)\right\},
$$

then we obtain

$$
\max \left\{\left|F\left(z^{0}+t \mathbf{b}\right)\right|:|t|=\frac{2}{L\left(z^{0}\right)}\right\} \leq g_{z^{0}}\left(t_{2}\right) \leq g_{z^{0}}\left(t_{1}\right) \exp \{9 B / 2\} .
$$

Applying Cauchy's inequality and using that $t_{1} \in \gamma_{1}$ we obtain for all $j \in\{1, \ldots, p\}$

$$
\begin{gathered}
\left|\partial_{\mathbf{b}}^{j} F\left(z^{0}+t_{1} \mathbf{b}\right)\right| \leq j !\left(4 L\left(z^{0}\right)\right)^{j} \max \left\{\left|F\left(z^{0}+t \mathbf{b}\right):\right| t-t_{1} \mid=\frac{1}{4 L\left(z^{0}\right)}\right\} \\
\leq j !\left(4 L\left(z^{0}\right)\right)^{j} \max \left\{\left|F\left(z^{0}+t \mathbf{b}\right):\right| t-t_{0} \mid=\frac{1}{2 L\left(z^{0}\right)}\right\} \\
g_{z^{0}}\left(t_{1}\right) \leq p !(4)^{p} \max \left\{\left|F\left(z^{0}+t \mathbf{b}\right):\right| t-t_{0} \mid=\frac{1}{2 L\left(z^{0}\right)}\right\} .
\end{gathered}
$$

Therefore, from (5.3) it follows that

$$
\begin{aligned}
& \left|F\left(z^{0}+t_{2} \mathbf{b}\right)\right|=\max \left\{\left|F\left(z^{0}+t \mathbf{b}\right)\right|:|t|=\beta / L\left(z^{0}\right)\right\} \\
& \leq g_{z^{0}}\left(t_{2}\right) \leq g_{z^{0}}\left(t_{1}\right) \exp \{9 B / 2\} \leq p !(4)^{p} \exp \{9 B / 2\} \\
& \times \max \left\{\left|F\left(z^{0}+t \mathbf{b}\right)\right|:|t|=1 /\left(2 L\left(z^{0}\right)\right)\right\} .
\end{aligned}
$$

By Proposition 3.1 we conclude that the function $F$ has bounded $L$-index in the direction $\mathbf{b} \in \mathbb{C}^{n}$. Theorem 5.1 is proved. 
Using Theorem 5.1 we prove the following

Theorem 5.2. Let $L \in Q_{\mathbf{b}}^{n}$. A function $F \in \widetilde{\mathcal{H}}_{\mathbf{b}}^{n}$ has bounded L-index in the direction $\mathbf{b}$ if and only if there exist numbers $C \in(0,+\infty)$ and $N \in \mathbb{N}$ such that for all $z \in \mathbb{C}^{n}$

$$
\sum_{k=0}^{N} \frac{\left|\partial_{\mathbf{b}}^{k} F(z)\right|}{k ! L^{k}(z)} \geq C \sum_{k=N+1}^{\infty} \frac{\left|\partial_{\mathbf{b}}^{k} F(z)\right|}{k ! L^{k}(z)} .
$$

Proof. Proof of this theorem is similar to proof of its analogs for entire functions of bounded $L$-index in direction [3] and for entire functions of bounded $l$-index $[25]$.

Let $0<\theta<1$. If the function $F$ is of bounded $L$-index in the direction b, then by Lemma $2.1 F$ is also of bounded $L^{*}$-index in the direction $\mathbf{b}$, where $L^{*}(z)=\theta L(z)$. Denote $N^{*}=N_{\mathbf{b}}\left(F, L_{*}\right)$ and $N=N_{\mathbf{b}}(F, L)$. Thus,

$$
\begin{aligned}
\max \left\{\frac{\left|\partial_{\mathbf{b}}^{k} F(z)\right|}{k ! L^{k}(z)}\right. & \left.: 0 \leq k \leq N^{*}\right\}=\max \left\{\frac{\left|\partial_{\mathbf{b}}^{k} F(z)\right|}{k ! L_{*}^{k}(z)} \theta^{k}: 0 \leq k \leq N^{*}\right\} \\
\geq & \theta^{N^{*}} \max \left\{\frac{\left|\partial_{\mathbf{b}}^{k} F(z)\right|}{k ! L_{*}^{k}(z)}: 0 \leq k \leq N^{*}\right\} \\
& \geq \theta^{N^{*}} \frac{\left|\partial_{\mathbf{b}}^{j} F(z)\right|}{j ! L_{*}^{j}(z)}=\theta^{N^{*}-j} \frac{\left|\partial_{\mathbf{b}}^{j} F(z)\right|}{j ! L^{j}(z)}
\end{aligned}
$$

for all $j \geq 0$ and

$$
\begin{aligned}
& \sum_{j=N^{*}+1}^{\infty} \frac{\left|\partial_{\mathbf{b}}^{j} F(z)\right|}{j ! L^{j}(z)} \leq \max \left\{\frac{\left|\partial_{\mathbf{b}}^{k} F(z)\right|}{k ! L^{k}(z)}: 0 \leq k \leq N^{*}\right\} \sum_{j=N^{*}+1}^{\infty} \theta^{j-N^{*}} \\
& =\frac{\theta}{1-\theta} \max \left\{\frac{\left|\partial_{\mathbf{b}}^{k} F(z)\right|}{k ! L^{k}(z)}: 0 \leq k \leq N^{*}\right\} \leq \frac{\theta}{1-\theta} \sum_{k=0}^{N^{*}} \frac{\left|\partial_{\mathbf{b}}^{k} F(z)\right|}{k ! L^{k}(z)}
\end{aligned}
$$

i.e. we obtain (5.4) with $N=N^{*}$ and $C=\frac{1-\theta}{\theta}$.

Now we prove the sufficiency. From (5.4) we obtain

$$
\begin{gathered}
\frac{\left|\partial_{\mathbf{b}}^{N+1} F(z)\right|}{(N+1) ! L^{N+1}(z)} \leq \sum_{k=N+1}^{\infty} \frac{\left|\partial_{\mathbf{b}}^{k} F(z)\right|}{k ! L^{k}(z)} \leq \frac{1}{C} \sum_{k=0}^{N} \frac{\left|\partial_{\mathbf{b}}^{k} F(z)\right|}{k ! L^{k}(z)} \\
\leq \frac{N+1}{C} \max \left\{\frac{\left|\partial_{\mathbf{b}}^{k} F(z)\right|}{k ! L^{k}(z)}: 0 \leq k \leq N\right\}
\end{gathered}
$$

Applying Theorem 5.1, we obtain the desired conclusion.

Using Theorems 2.2 and 5.1 we obtain this corollary. 
Corollary 5.1. Let $L \in Q_{\mathbf{b}}^{n}, F \in \mathcal{H}_{\mathbf{b}}^{n},(\forall p \in \mathbb{N}) \partial_{\mathbf{b}}^{p} F \in \mathcal{H}_{\mathbf{b}}^{n}$, G be a bounded domain in $\mathbb{C}^{n}$ such that $\forall z \in \bar{G} F(z+t \mathbf{b}) \not \equiv 0$. The function $F$ has bounded L-index in the direction $\mathbf{b}$ if and only if there exist $p \in \mathbb{Z}_{+}$ and $C>0$ such that for all $z \in \mathbb{C}^{n} \backslash G$ the inequality (5.1) holds.

\section{Functions having bounded value $L$-distribution in direction}

An entire function $f(z)(z \in \mathbb{C})$ is said ot be of bounded value distribution $[16,18,24]$, if there exist $p \geq 0, R>0$ such that the equation $f(z)=w$ has at least $p$ roots in any disc of radius $R$.

One of the remarkable properties generating big interest to functions of bounded index is the following fact proved by W. Hayman [18]: an entire function has bounded value distribution if and only if it derivative has bounded index. Leter, there was introduced a concept of entire function of bounded value $l$-distribution [19], and this property was generalized for entire functions of bounded $l$-index [27]. For entire bivariate functions of bounded index in joint variables similar results are partially obtained in [23].

Definition 6.1. Function $F \in \widetilde{\mathcal{H}}_{\mathbf{b}}^{n}$ is said to be of bounded value $L$ distribution in a direction $\mathbf{b}$ if for all $p \in \mathbb{N} \forall w \in \mathbb{C} \forall z_{0} \in \mathbb{C}^{n}$ such that $F\left(z^{0}+t \mathbf{b}\right) \not \equiv w$, the inequality holds $n\left(\frac{1}{L\left(z^{0}\right)}, z^{0}, \frac{1}{F-w}\right) \leq p$, i.e. the equation $F\left(z^{0}+t \mathbf{b}\right)=w$ has at most $p$ solutions in the disc $\{t$ : $\left.|t| \leq \frac{1}{L\left(z^{0}\right)}\right\}$. In other words, the function $F\left(z^{0}+t \mathbf{b}\right)$ is p-valent in $\left\{t:|t| \leq \frac{1}{L\left(z^{0}\right)}\right\}$.

The corresponding Sheremeta result [27] we will generalize for the functions from the class $\widetilde{\mathcal{H}}_{\mathrm{b}}^{n}$, which have bounded value $L$-distribution in direction $\mathbf{b}$.

Proposition 6.1. Let $L \in Q_{\mathrm{b}}^{n}$. An entire function $F \in \widetilde{\mathcal{H}}_{\mathrm{b}}^{n}$ is a function of bounded value L-distribution in the direction $\mathbf{b}$ if and only if the directional derivative $\partial_{\mathbf{b}} F \in \widetilde{\mathcal{H}}_{\mathbf{b}}^{n}$ has bounded L-index in the same direction b.

Proof. This is similar to the proof of the corresponding proposition for analytic functions in the unit ball [2].

Suppose that $F$ is of bounded value $L$-distribution in direction $\mathbf{b}$, i.e. for all $z^{0} \in \mathbb{C}^{n}$ such that $F\left(z^{0}+t \mathbf{b}\right) \not \equiv$ const the function $F\left(z^{0}+t \mathbf{b}\right)$ is $p$-valent in every disc $\left\{t:|t| \leq \frac{1}{L\left(z^{0}\right)}\right\}$.

To prove the proposition we need the following proposition from $[26$, p. 48, Theorem 2.8]. 
Theorem 6.1 ([26]). Let $D_{0}=\left\{t:\left|t-t_{0}\right|<R\right\}, 0<R<\infty$. If analytic in $D_{0}$ function $f$ is p-valent in $D_{0}$, then for $j>p$

$$
\frac{\left|f^{(j)}\left(t_{0}\right)\right|}{j !} R^{j} \leq(A j)^{2 p} \max \left\{\frac{\left|f^{(k)}\left(t_{0}\right)\right|}{k !} R^{k}: 1 \leq k \leq p\right\},
$$

where $A=\sqrt[2 p]{\frac{p+2}{2}} \sqrt{8 e^{\pi^{2}}}$.

By Theorem 6.1 inequality (6.1) holds with $R=\frac{1}{L\left(z^{0}\right)}$ for the function $F\left(z^{0}+t \mathbf{b}\right)$, as a function of single variable $t \in \mathbb{C}$ for every fixed $z^{0} \in \mathbb{C}^{n}$. Then it is easy to deduce that for every $m \in \mathbb{N}$ the following equality $g_{z^{0}}^{(p)}(t)=\partial_{\mathbf{b}}^{p} F\left(z^{0}+t \mathbf{b}\right)$ holds. Take $j=p+1$ and $t_{0}=0$ in Theorem 6.1. From (6.1) it follows

$$
\begin{gathered}
\frac{\left|\partial_{\mathbf{b}}^{p+1} F\left(z^{0}\right)\right|}{(p+1) ! L^{p+1}\left(z_{0}\right)} \leq(A(p+1))^{2 p} \max \left\{\frac{\left|\partial_{\mathbf{b}}^{k} F\left(z^{0}\right)\right|}{k ! L^{k}\left(z_{0}\right)}: 1 \leq k \leq p\right\} \Rightarrow \\
\frac{\left|\partial_{\mathbf{b}}^{p+1} F\left(z^{0}\right)\right|}{L^{p+1}\left(z_{0}\right)} \leq(p+1) !(A(p+1))^{2 p} \max \left\{\frac{\left|\partial_{\mathbf{b}}^{k} F\left(z^{0}\right)\right|}{L^{k}\left(z_{0}\right)}: 1 \leq k \leq p\right\} \\
\quad \times \max \left\{\frac{1}{k !}: 1 \leq k \leq p\right\} \Rightarrow \\
\frac{\left|\partial_{\mathbf{b}}^{p} \partial_{\mathbf{b}} F\left(z^{0}\right)\right|}{L^{p}\left(z_{0}\right)} \leq L\left(z^{0}\right) \cdot(p+1) ! A^{2 p}(p+1)^{2 p} \max \left\{\frac{\left|\partial_{\mathbf{b}}^{k-1} \partial_{\mathbf{b}} F\left(z^{0}\right)\right|}{L^{k}\left(z_{0}\right)}:\right. \\
0 \leq k-1 \leq p-1\} \Rightarrow \frac{\left|\partial_{\mathbf{b}}^{p} \partial_{\mathbf{b}} F\left(z^{0}\right)\right|}{L^{p}\left(z_{0}\right)}: \\
\leq(p+1) ! A^{2 p}(p+1)^{2 p} \max \left\{\frac{\left|\partial_{\mathbf{b}}^{k-1} \partial_{\mathbf{b}} F\left(z^{0}\right)\right|}{L^{k-1}\left(z_{0}\right)}: 0 \leq k-1 \leq p-1\right\} .
\end{gathered}
$$

Now we need analog of Hayman's Theorem proved above. Thus, for $\partial_{\mathbf{b}} F$ inequality (5.1) holds with $p-1$ instead $p$ and with $C=(p+1) ! A^{2 p}(p+$ $1)^{2 p}$. In Theorem 6.1 the constant $A \geq \max _{j>p} \frac{p+2}{2}\left(8 e^{\pi^{2}}\right)^{p}\left(1-\frac{1}{j}\right)^{j}$ does not depend from $z^{0}$, because the parameter $p$ is independent of $z^{0}$. Hence, the quantity $C=(p+1) ! A^{2 p}(p+1)^{2 p}$ does not depend of $z^{0}$. Therefore by Theorem 5.1 the function $\partial_{\mathbf{b}} F$ has bounded $L$-index in the direction b.

Conversely, let $\partial_{\mathbf{b}} F$ be a function of bounded $L$-index in the direction b. By Theorem 5.1 there exist $p \in \mathbb{Z}_{+}$and $C \geq 1$ such that for every 
$z \in \mathbb{C}^{n}$ the following inequality holds

$$
\frac{\left|\partial_{\mathbf{b}}^{p+1} F(z)\right|}{L^{p+1}(z)} \leq C \max \left\{\frac{\left|\partial_{\mathbf{b}}^{k} F(z)\right|}{L^{k}(z)}: 1 \leq k \leq p\right\} .
$$

Let us consider a disc $K_{0}=\left\{t \in \mathbb{C}:|t| \leq \frac{1}{L\left(z^{0}\right)}\right\}, z^{0} \in \mathbb{C}^{n}$.

One should observe that if $L \in Q_{\mathbf{b}}^{n}, z^{0} \in \mathbb{C}^{n}$ then for all $r>0$ the inequality $|t| \leq \frac{r}{L\left(z^{0}\right)}$ and definition of class $Q_{\mathbf{b}}^{n}$ yield

$$
L\left(z^{0}\right) / \lambda_{\mathbf{b}}(r) \leq L\left(z^{0}+t \mathbf{b}\right) \leq \lambda_{\mathbf{b}}(r) L\left(z^{0}\right) .
$$

Now from (6.2) and (6.3) for $z=z^{0}+t \mathbf{b}, t \in K$ one has

$$
\begin{gathered}
\frac{\left|\partial_{\mathbf{b}}^{p+1} F\left(z^{0}+t \mathbf{b}\right)\right|}{(p+1) !\left(C \lambda_{\mathbf{b}}(1) L\left(z^{0}\right)\right)^{p+1}} \leq \max \left\{\frac{\left|\partial_{\mathbf{b}}^{k} F\left(z^{0}+t \mathbf{b}\right)\right|}{k !} \frac{1}{\left(C \lambda_{\mathbf{b}}(1) L\left(z^{0}\right)\right)^{k}}\right. \\
\left.\quad \times\left(\frac{L\left(z^{0}+t \mathbf{b}\right)}{C \lambda_{\mathbf{b}}(1) L\left(z^{0}\right)}\right)^{p+1-k}: 1 \leq k \leq p\right\} \leq \frac{C}{p+1} \\
\quad \times \max _{1 \leq k \leq p}\left\{\frac{\left|\partial_{\mathbf{b}}^{k} F\left(z^{0}+t \mathbf{b}\right)\right|}{k !} \frac{1}{\left(C \lambda_{\mathbf{b}}(1) L\left(z^{0}\right)\right)^{k}} \frac{1}{C{ }^{p+1-k}}\right\} \\
\leq \max \left\{\frac{\left|\partial_{\mathbf{b}}^{k} F\left(z^{0}+t \mathbf{b}\right)\right|}{k !} \frac{1}{\left(C \lambda_{\mathbf{b}}(1) L\left(z^{0}\right)\right)^{k}}: 1 \leq k \leq p\right\}
\end{gathered}
$$

To prove the proposition we need such a statement from [26, p. 44, Theorem 2.7].

Theorem 6.2 ( $\left[26\right.$, p.44, Theorem 2.7]). Let $D_{0}=\left\{t \in \mathbb{C}:\left|t-t_{0}\right|<\right.$ $R\}, 0<R<+\infty$, and $f(t)$ is an analytic function in $D_{0}$. If for all $t \in D_{0}$

$$
\left(\frac{R}{2}\right)^{p+1} \frac{\left|f^{(p+1)}(t)\right|}{(p+1) !} \leq \max \left\{\left(\frac{R}{2}\right)^{k} \frac{\left|f^{(k)}(t)\right|}{k !}: 1 \leq k \leq p\right\},
$$

then $f(t)$ is p-valent in $\left\{t \in \mathbb{C}:\left|t-t_{0}\right| \leq \frac{R}{25 \sqrt{p+1}}\right\}$, i.e., $f(t)$ attains every value at more $p$ times.

From inequality (6.4) it follows inequality (6.5) with $R=\frac{2}{C \lambda_{\mathbf{b}}(1) L\left(z^{0}\right)}$ and $t_{0}=0$. By Theorem 6.2 the function $F\left(z^{0}+t \mathbf{b}\right)$ is $p$-valent in the $\operatorname{disc}\left\{t \in \mathbb{C}:|t| \leq \frac{\rho}{L\left(z^{0}\right)}\right\}, \rho=\frac{2}{25 C \lambda_{\mathbf{b}}(1) \sqrt{p+1}}$.

Let $t_{j}$ be an arbitrary point in $K_{0}$ and $K_{j}^{*}=\left\{t \in \mathbb{C}:\left|t-t_{i}\right| \leq\right.$ $\left.\frac{\rho}{L\left(z^{0}+t_{j} \mathbf{b}\right)}\right\}$. Since by definition of class $Q_{\mathbf{b}}^{n} \quad L\left(z^{0}+t_{j} \mathbf{b}\right) \leq \lambda_{\mathbf{b}}(1) L\left(z^{0}\right)$, one has $K_{j}=\left\{t \in \mathbb{C}:\left|t-t_{j}\right| \leq \frac{\rho}{\lambda_{\mathbf{b}}(1) L\left(z^{0}\right)}\right\} \subset K_{j}^{*}$. We will repeat 
the similar considerations for the set $\left\{t \in \mathbb{C}:\left|t-t_{j}\right| \leq \frac{1}{L\left(z^{0}+t_{j} \mathbf{b}\right)}\right\}$. As a consequence, we deduce that $F\left(z^{0}+t \mathbf{b}\right)$ is $p$-valent in $K_{j}^{*}$. But $K_{j} \subset K_{j}^{*}$, then $F\left(z^{0}+t \mathbf{b}\right)$ is $p$-valent in $K_{j}$.

Finally, we note that every closed disc of radius $R_{*}$ can be covered by a finite number $m_{*}$ of closed discs of radius $\rho_{*}<R_{*}$ with the centers in the disc. Moreover, $m_{*}<B_{*}\left(R_{*} / \rho_{*}\right)^{2}$, where $B_{*}>0$ is an absolute constant. Hence, $K_{0}$ can be covered finite number $m$ of discs $K_{j}$, where $m \leq 625 B^{*}(p+1) C^{2}\left(\lambda_{\mathbf{b}}(1)\right)^{2} / 4$. Since the function $F\left(z^{0}+t \mathbf{b}\right)$ in $K_{j}$ is $p$-valent, it is $m p$-valent in $K_{0}$.

In view of arbitrariness of $z^{0}$, the statement is proved.

\section{Existence theorem for functions of bounded $L$-index in direction}

For the one-dimensional case, some time ago mathematicians were interested in the following two problems: the problem of the existence of an entire function of bounded $l$-index for a given $l$, and the problem of the existence of a function $l$ for a given entire function $f$ such that $f$ is of bounded $l$-index $[12,13,17,29]$. It is clear that similar problems can be posed for the multidimensional case $[5,7]$.

We note that the solution of the first problem for the one-dimensional case is given by a canonical product. The solution of the first problem in the multidimensional case also exists in the class of canonical products with "planar" zeros.

In particular, the following proposition is true.

We consider the function $F\left(z^{0}+t \mathbf{b}\right)$ where $z^{0} \in \mathbb{C}^{n}$ is fixed. If $F\left(z^{0}+t \mathbf{b}\right) \not \equiv 0$, then we denote by $p_{\mathbf{b}}\left(z^{0}+a_{k}^{0} \mathbf{b}\right)$ the multiplicity of the zero $a_{k}^{0}$ of the function $F\left(z^{0}+t \mathbf{b}\right)$. If $F\left(z^{0}+t \mathbf{b}\right) \equiv 0$ for some $z^{0} \in \mathbb{C}^{n}$, then we put $p_{\mathbf{b}}\left(z^{0}+t \mathbf{b}\right)=-1$.

Theorem 7.1. In order that for a function $F \in \widetilde{\mathcal{H}}_{\mathrm{b}}^{n}$ there exist a positive continuous function $L(z)$ such that $F(z)$ is of bounded $L$-index in the direction $\mathbf{b}$ it is necessary and sufficient that $\exists p \in \mathbb{Z}_{+} \forall z^{0} \in \mathbb{C}^{n} \forall k$ $p_{\mathbf{b}}\left(z^{0}+a_{k}^{0} \mathbf{b}\right) \leq p$.

Proof. Necessity. To simplify the notation we denote everywhere in the proof $p_{k}^{0} \equiv p_{\mathbf{b}}\left(z^{0}+a_{0}^{k} \mathbf{b}\right)$. One can prove the necessity using the definition of bounded $L$-index in direction. Indeed, assume on the contrary that $\forall p \in \mathbb{Z}_{+} \exists z^{0} \exists k p_{k}^{0}>p$. It means that $\partial_{\mathbf{b}}^{p_{k}^{0}} F\left(z^{0}+a_{k}^{0} \mathbf{b}\right) \neq 0$ and $\partial_{\mathbf{b}}^{j} F\left(z^{0}+a_{k}^{0} \mathbf{b}\right)=0$ for all $j \in\left\{1, \ldots, p_{k}^{0}-1\right\}$. Therefore, the $L$-index in the direction $b$ at the point $z^{0}+a_{k}^{0} \mathbf{b}$ is not less than $p_{k}^{0}>p$

$$
N_{\mathbf{b}}\left(F, L, z^{0}+a_{k}^{0} \mathbf{b}\right)>p .
$$


If $p \rightarrow+\infty$, then we obtain that $N_{\mathbf{b}}\left(F, L, z^{0}+a_{k}^{0} \mathbf{b}\right) \rightarrow+\infty$. But this contradicts the boundedness of $L$-index in the direction $\mathbf{b}$ of the function $F$.

Sufficiency. If $F\left(z^{0}+t \mathbf{b}\right) \equiv 0$ for some $z^{0} \in \mathbb{C}^{n}$, then inequality (1.1) is obvious.

Let $p$ be the least integer such that $\forall z^{0} \in \mathbb{C}^{n} F\left(z^{0}+t \mathbf{b}\right) \not \equiv 0$, and $\forall k$ $p_{k}\left(z^{0}\right) \leq p$. For any point $z \in \mathbb{C}^{n}$ we choose $z^{0} \in \mathbb{C}^{n}$ and $t_{0} \in \mathbb{C}$ so that $z=z^{0}+t_{0} \mathbf{b}$ and the point $z^{0}$ lies on the hyperplane $\langle z, m\rangle=1$, where $\langle\mathbf{b}, m\rangle=1$ (actually it is sufficient that $\langle\mathbf{b}, m\rangle \neq 0$, i.e. the hyperplane is not parallel to $\mathbf{b})$. Therefore, $t_{0}=\langle z, m\rangle-1, z^{0}=z-(\langle z, m\rangle-1) \mathbf{b}$. We put $K_{R}=\{t \in \mathbb{C}: \max \{0, R-1\} \leq|t| \leq R+1\}$ for all $R \geq 0$ and

$$
m_{1}\left(z^{0}, R\right)=\min _{a_{k}^{0} \in K_{R}} \max _{0 \leq s \leq p}\left\{\frac{\left|\partial_{\mathbf{b}}^{s} F\left(z^{0}+a_{k}^{0} \mathbf{b}\right)\right|}{s !}\right\} .
$$

Since $F$ is a slice entire function, there exists $\varepsilon=\varepsilon\left(z^{0}, R\right)>0$ such that

$$
\frac{\left|\partial_{\mathbf{b}}^{s_{0}} F\left(z^{0}+t \mathbf{b}\right)\right|}{s_{0} !} \geq \frac{m_{1}\left(z^{0}, R\right)}{2}
$$

for some $s_{0}=s\left(a_{k}^{0}\right) \in\{0, \ldots, p\}$ and for all $t \in K_{R} \cap\left\{t \in \mathbb{C}:\left|t-a_{k}^{0}\right|<\right.$ $\left.\varepsilon\left(R, z^{0}\right)\right\}$ and for all $k$. We denote $G_{\varepsilon}^{0}=\bigcup_{a_{k}^{0} \in K_{R}}\left\{t \in \mathbb{C}:\left|t-a_{k}^{0}\right|<\varepsilon\right\}$, $m_{2}\left(z^{0}, R\right)=\min \left\{\left|F\left(z^{0}+t \mathbf{b}\right)\right|:|t| \leq R+1, t \notin G_{\varepsilon}^{0}\right\}$,

$$
Q\left(R, z^{0}\right)=\min \left\{\frac{m_{1}\left(R, z^{0}\right)}{2}, m_{2}\left(R, z^{0}\right), 1\right\} .
$$

We take $R=\left|t_{0}\right|$. Then at least one of the numbers $\left|F\left(z^{0}+t_{0} \mathbf{b}\right)\right|$, $\left|\partial_{\mathbf{b}} F\left(z^{0}+t_{0} \mathbf{b}\right)\right|, \ldots, \frac{\left|\partial_{\mathbf{b}}^{p} F\left(z^{0}+t_{0} \mathbf{b}\right)\right|}{p !}$ is not less than $Q\left(R, z^{0}\right)$ (respectively, for $t_{0} \in G_{\varepsilon}^{0} \quad \frac{\left.\mid \partial_{\mathbf{b}}^{s_{0}} F\left(z^{0}+t_{0}\right) \mathbf{b}\right) \mid}{p_{k}^{0} !}$ and for $\left.t \notin G_{\varepsilon} \quad\left|F\left(z^{0}+t_{0} \mathbf{b}\right)\right|\right)$.

Hence,

$$
\max \left\{\frac{\left|\partial_{\mathbf{b}}^{j} F\left(z^{0}+t_{0} \mathbf{b}\right)\right|}{j !}: 0 \leq j \leq p\right\} \geq Q\left(R, z^{0}\right) .
$$

On the other hand, for $\left|t_{0}\right|=R$ and $j \geq p+1$ Cauchy's inequality is valid

$$
\begin{gathered}
\frac{\left|\partial_{\mathbf{b}}^{j} F\left(z^{0}+t_{0} \mathbf{b}\right)\right|}{j !} \leq \frac{1}{2 \pi} \int_{\left|\tau-t_{0}\right|=1} \frac{\left|F\left(z^{0}+\tau \mathbf{b}\right)\right|}{\left|\tau-t_{0}\right|^{j+1}}|d \tau| \\
\leq \max \left\{\left|F\left(z^{0}+\tau \mathbf{b}\right)\right|:|\tau| \leq R+1\right\} .
\end{gathered}
$$


We choose a positive continuous function $L(z)$ such that

$$
L\left(z^{0}+t_{0} \mathbf{b}\right) \geq \max \left\{\frac{\max \left\{\left|F\left(z^{0}+t \mathbf{b}\right)\right|:|\tau| \leq R+1\right\}}{Q\left(R, z^{0}\right)}, 1\right\} .
$$

From (7.1) and (7.2) with $\left|t_{0}\right|=R$ and $j \geq p+1$ we obtain

$$
\begin{gathered}
\frac{\frac{\left|\partial_{\mathbf{b}}^{j} F\left(z^{0}+t_{0} \mathbf{b}\right)\right|}{j ! L^{j}\left(z^{0}+t_{0} \mathbf{b}\right)}}{\max \left\{\frac{\left|\partial_{\mathbf{b}}^{k} F\left(z^{0}+t_{0} \mathbf{b}\right)\right|}{k ! L^{k}\left(z^{0}+t_{0} \mathbf{b}\right)}: 0 \leq k \leq p\right\}} \leq \frac{L^{-j}\left(z^{0}+t \mathbf{b}\right)}{Q\left(R, z^{0}\right) L^{-p}\left(z^{0}+t \mathbf{b}\right)} \\
\times \max \left\{\left|F\left(z^{0}+t \mathbf{b}\right)\right|:|\tau| \leq R+1\right\} \leq L^{p+1-j}\left(z^{0}+t \mathbf{b}\right) \leq 1 .
\end{gathered}
$$

Since $z=z^{0}+t_{0} \mathbf{b}$, we have

$$
\frac{\left|\partial_{\mathbf{b}}^{j} F(z)\right|}{j ! L^{j}(z)} \leq \max \left\{\frac{\left|\partial_{\mathbf{b}}^{k} F(z)\right|}{k ! L^{k}(z)}: 0 \leq k \leq p\right\} .
$$

In view of arbitrariness of $z$ the function $F$ has bounded $L$-index in the direction $\mathbf{b}$.

\section{References}

[1] A. I. Bandura, O. B. Skaskiv, Slice holomorphic functions in several variables having bounded L-index in direction, submitted to Axioms.

[2] A. I. Bandura, Analytic functions in the unit ball of bounded value L-distribution in a direction // Mat. Stud., 49 (2018), No. 1, 75-79.

[3] A. I. Bandura, Product of two entire functions of bounded L-index in direction is a function with the same class // Bukovyn. Mat. Zh., 4 (2016), No. 1-2, 8-12.

[4] A. I. Bandura, O. B. Skaskiv, Sufficient conditions of boundedness of L-index and analog of Hayman's Theorem for analytic functions in a ball // Stud. Univ. Babeş-Bolyai Math., 63 (2018), No. 4, 483-501.

[5] A. I. Bandura, O. B. Skaskiv, Analytic functions in the unit ball of bounded Lindex in joint variables and of bounded L-index in direction: a connection between these classes // Demonstr. Math., 52 (2019), No. 1, 82-87.

[6] A. I. Bandura, O. B. Skaskiv, Entire functions of bounded L-index in direction // Mat. Stud., 27 (2007), No. 1, 30-52.

[7] A. I. Bandura, O. B. Skaskiv, Boundedness of L-index in direction of functions of the form $f(\langle z, m\rangle)$ and existence theorems // Mat. Stud., 41 (2014), No. 1, $45-52$.

[8] A. Bandura, O. Skaskiv, Entire functions of several variables of bounded index, Lviv, Publisher I. E. Chyzhykov, 2016. 
[9] A. Bandura, N. Petrechko, O. Skaskiv, Maximum modulus in a bidisc of analytic functions of bounded $\mathbf{L}$-index and an analogue of Hayman's theorem // Mat. Bohemica, 143 (2018), No. 4, 339-354.

[10] A. Bandura, O. Skaskiv, Directional logarithmic derivative and the distribution of zeros of an entire function of bounded L-index along the direction // Ukr. Math. J., 69 (2017), No. 3, 500-508.

[11] A. I. Bandura, Some improvements of criteria of L-index boundedness in direction // Mat. Stud., 47 (2017), No. 1, 27-32.

[12] M. T. Bordulyak, M. M. Sheremeta, On the existence of entire functions of bounded l-index and l-regular growth // Ukrainian Math. J., 48 (1996), No. 9, $1322-1340$.

[13] M. T. Bordulyak, A proof of Sheremeta conjecture concerning entire function of bounded l-index // Mat. Stud., 12 (1999), No. 1, 108-110.

[14] G. H. Fricke, Functions of bounded index and their logarithmic derivatives // Math. Ann., 206 (1973), 215-223.

[15] G. H. Fricke, Entire functions of locally slow growth // J. Anal. Math., 28 (1975), No. 1, 101-122.

[16] G. H. Fricke, S. M. Shah, On bounded value distribution and bounded index // Nonlinear Anal., 2 (1978), No. 4, 423-435.

[17] A. A. Goldberg, M. N. Sheremeta, Existence of an entire transcendental function of bounded l-index // Math. Notes, 57 (1995), No. 1, 88-90.

[18] W. K. Hayman, Differential inequalities and local valency // Pacific J. Math., 44 (1973), No. 1, 117-137.

[19] A. D. Kuzyk, M. N. Sheremeta, Entire functions of bounded l-distribution of values // Math. Notes, 39 (1986), No. 1, 3-8.

[20] A. D. Kuzyk, M. N. Sheremeta, On entire functions, satisfying linear differential equations // Diff. equations, 26 (1990), No. 10, 1716-1722.

[21] B. Lepson, Differential equations of infinite order, hyperdirichlet series and entire functions of bounded index // Proc. Sympos. Pure Math., 2 (1968), 298-307.

[22] J. J. Macdonnell, Some convergence theorems for Dirichlet-type series whose coefficients are entire functions of bounded index, Doctoral dissertation, Catholic University of America, Washington, 1957.

[23] F. Nuray, R. F. Patterson, Multivalence of bivariate functions of bounded index // Le Matematiche, 70 (2015), No. 2, 225-233.

[24] S. Shah, Entire functions of bounded value distribution and gap power series, In: P. Erdős, L. Alpár, G. Halász, A. Sárközy (eds.) Studies in Pure Mathematics To the Memory of Paul Turán, pp. 629-634, Birkhäuser Basel (1983). 
[25] M. N. Sheremeta, A. D. Kuzyk, Logarithmic derivative and zeros of an entire function of bounded l-index // Sib. Math. J., 33 (1992), No. 2, 304-312.

[26] M. Sheremeta, Analytic functions of bounded index, Lviv, VNTL Publishers, 1999.

[27] M. N. Sheremeta, An l-index and an l-distribution of the values of entire functions // Soviet Math. (Iz. VUZ), 34 (1990), No. 2, 115-117.

[28] M. M. Sheremeta, Generalization of the Fricke theorem on entire functions of finite index // Ukrainian Math. J., 48 (1996), No. 3, 460-466.

[29] M. M. Sheremeta, Remark to existence theorem for entire function of bounded l-index // Mat. Stud., 13 (2000), No. 1, 97-99.

[30] M. M. Sheremeta, M. T. Bordulyak, Boundedness of the l-index of Laguerre-Polya entire functions // Ukr. Math. J., 55 (2003), No. 1, 112-125.

[31] S. Strelitz, Asymptotic properties of entire transcendental solutions of algebraic differential equations // Contemp. Math., 25 (1983), 171-214.

\section{CONTACT INFORMATION}

Andriy Bandura Ivano-Frankivsk National

Technical University of Oil and Gas,

Ivano-Frankivsk, Ukraine

E-Mail: andriykopanytsia@gmail.com

\section{Oleh Skaskiv}

Ivan Franko National University of Lviv,

Lviv, Ukraine

E-Mail: olskask@gmail.com 